\title{
Interventions for Improving Young Children's Dietary Intake through Early Childhood Settings: A Systematic Review
}

\author{
Lucinda K. Bell and Rebecca K. Golley*
}

\author{
Sansom Institute for Health Research, University of South Australia, Adelaide 5001, Australia
}

\begin{abstract}
Early childhood settings (ECS) offer a unique opportunity to intervene to improve children's nutrition. This paper reviews the literature on early childhood setting interventions that aim to improve children's dietary intake. Environmental and individual determinants of children's dietary intakes were also investigated. Prospective intervention studies targeting centres, staff, parents/caregivers or children, were reviewed. Methodological quality was assessed. Twenty six studies (14 weak, 12 moderate quality) were included. Interventions were delivered primarily via training workshops and/or written materials. Study findings favoured intervention effectiveness in 23 studies. Improvements were seen in children's intake for 8 out of 11 studies assessing dietary intake outcomes. Small increases in fruit and vegetable consumption were observed in five studies. Most studies measuring parental or centre food provision observed postintervention improvements across a number of food groups, including fruit, vegetables, whole grains and sweetened beverages. Significant improvements in child, parent and/or staff knowledge, attitudes or behaviours were observed consistently across studies. For those studies that included a comparison group, these improvements were observed only in the intervention group. ECS interventions can achieve changes in children's dietary intake and associated socioenvironmental- determinants, although the quality of current research limits confidence in study findings. Future intervention development needs to carefully consider the behavioural targets, modifiable determinants and utilise ageappropriate and effective behaviour change theory, in addition to inclusion of dietary intake outcomes.
\end{abstract}

Keywords: Early childhood settings, child care, preschool, nutrition, diet, review.

\section{INTRODUCTION}

Early life diet is a modifiable exposure which influences children's health and development [1, 2]. The diet provided to children in the first years of life changes from milk as the sole source of nutrition, to the introduction of foods and beverages that expand in variety, texture and appearance $[3,4]$. This is the most rapid change in diet over the life course and is the developmental period when dietary preferences and habits are established [5, 6]. This highlights the importance of providing young children with healthy food and eating opportunities across the range of settings where they spend their time, including their homes and other places of care [7].

With increasing numbers of mothers of young children re-entering the workforce, children are spending a significant proportion of their time in early childhood settings (ECS) such as child care and preschool. In the US, approximately $80 \%$ of preschoolaged children with employed mothers are in some form of centre-based care [8]. In Australia, around $50 \%$ of 23 year olds spend $20-30$ hours per week in formal care settings, with over $95 \%$ of $4-5$ year olds attending an ECS [9]. Given their high utilisation ECS are a potentially useful avenue for interventions.

*Address correspondence to this author at the Sansom Institute for Health Research, University of South Australia, GPO Box 2471, Adelaide 5001, Australia; Tel: (+61 8) 8302 2440; E-mail: rebecca.golley@unisa.edu.au
ECS commonly provide children with food while in care, potentially accounting for approximately $40-60 \%$ of their daily food intake [10]. Alternatively parents provide their child's food for the day [11], often guided by ECS food and nutrition policies. Previous research has shown that children's food intake while in ECS is not consistent with dietary recommendations, particularly for foods such as vegetables, fruit, whole grains and meat [12-14]. More broadly, poor dietary habits are already common in young children, with high intakes of discretionary foods at the expense of foods such as fruit, vegetables and whole grains $[15,16]$. Suboptimal dietary intakes in early life are known to track into child- and adult-hood [17] and impact on health and development [15]. ECS offer a unique opportunity to intervene early to improve children's nutrition and reduce the risk of short- and long-term consequences.

Despite the increasing understanding of ECS potential to improve children's nutrition, health and development (e.g. diet quality, calcium intake, weight status, cardiovascular risk factors, readiness to learn), recent reviews have focused narrowly on ECS as a setting for obesity prevention [18, 19]. A greater understanding of the how ECS interventions can most effectively improve children's dietary intake more broadly (i.e. beyond weight-related factors) is needed. Therefore this article reviews studies evaluating the effectiveness of ECS nutrition promotion interventions 
on children's (aged 0-5 years) dietary intake (primary outcome) whilst in care. As environmental and individual factors such as centre's nutrition policies and practices, the food and beverages offered to children in ECS, and food or nutrition knowledge and attitudes influence children's dietary intakes [20], these outcomes were also investigated (secondary outcomes).

\section{METHODS}

\section{Criteria for Considering Studies for Review}

$\underline{\text { Inclusion Criteria }}$

\section{Types of Studies}

Prospective studies with or without a control or comparison group, evaluating the effectiveness of an intervention of any duration, with outcomes measured at baseline and post intervention.

\section{Types of Participants and Setting}

Intervention participants were ECS providers/staff, children (aged 0-5 years) or parents of children attending child care or family day care, preschool or nursery school.

\section{Types of Interventions}

Interventions that included a nutrition component targeting staff (e.g. staff training), children (e.g. a nutrition curriculum) or parents/caregivers (e.g. through education sessions or provision of resources) that aimed to influence children's nutritional intake. Participant involvement was defined by being able to identify exposure to the intervention

\section{Types of Outcomes Measures}

The primary outcome was children's dietary intake. Secondary outcomes were determinants of children's intake such as parent or staff knowledge, attitudes or behaviours, food availability, or the centre environment (e.g. centres' nutrition practices or policy). Although no criteria were set for outcomes to be measured using a validated tool, this was considered in the study quality assessment tool used (discussed below).

\section{Exclusion Criteria}

Studies were excluded if they were not applicable to the general population (for example preterm infants, behavioural/learning difficulties, disabilities and chronic conditions), not applicable to a high income country setting, with no full text article accessible in English.

\section{Search Method}

A three-step search strategy was undertaken to find published studies in the English language released up to June 2013 (Figure 1). In Stage 1 Cumulative Index to Nursing and Allied Health Literature (CINAHL), and Medline via PubMed were searched. A list of search terms and keywords were informed by relevant reviews $[7,18]$ and the review aim. The final search strategy (available from the authors) used the following keywords, tailored to each database:

1) Study participants: Child care/child day care/nursery school/preschool combined with staff/child/parent

2) Study type: Randomized controlled trial/ controlled clinical trial/ intervention

3) Study outcomes: diet/food/food intake/dietary intake.

Stage 2 involved identification of relevant articles, not captured in Stage 1, from a recent review [18]. In Stage 3, reference lists of all identified studies were searched for additional studies. No further searching of the grey literature was conducted.

Titles and abstracts of articles identified by the search were independently screened against the exclusion criteria by the first author (LB). Articles not meeting the eligibility criteria were excluded. Where it was unclear, the full text of the article was retrieved. All full-text articles included in the review were screened by the first author (LB) and any uncertainties were resolved by consensus with the second author (RG). Figure 1 summarises the review article selection.

\section{Data Extraction and Synthesis}

Data, including study characteristics, methodological quality and intervention content, from included studies was extracted by one author (LB) and verified by a second (RG). The methodological quality of studies was scored using the Effective Public Health Practice Project quality assessment tool [21]. In an assessment of 213 quality assessment tools, this tool was identified as useful for systematic reviews that evaluate randomized and non-randomized intervention studies [22]. Six quality components were scored (weak/moderate/strong): selection bias, study design and allocation bias, confounders, blinding, data collection methods (validity/reliability), withdrawals and dropouts. An overall quality rating was assigned; 
Potentially relevant studies identified and screened for retrieval $(\mathrm{n}=192)$

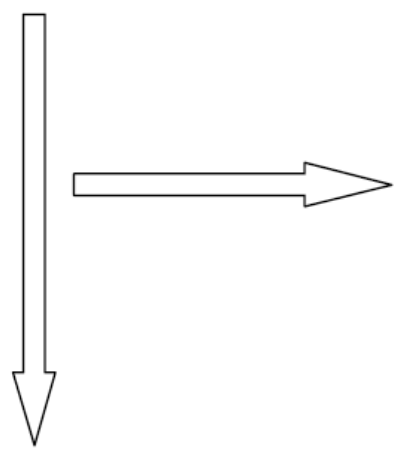

Studies retrieved for full text evaluation $(\mathrm{n}=21)$

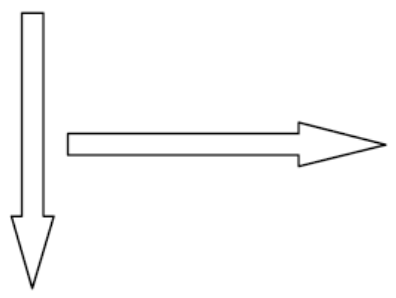

Included for review $(\mathrm{n}=13)$

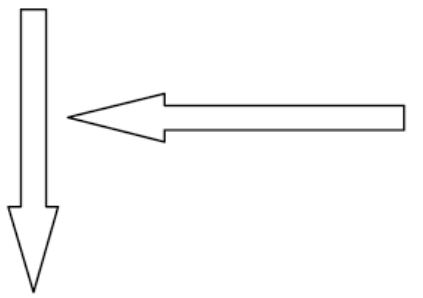

Additional studies identified from:

- Larson et al $2012(n=4)$

- Hand searching reference lists of included studies $(\mathrm{n}=6)$

- Knowledge of relevant articles in the literature $(\mathrm{n}=3)$

\section{Studies reviewed $(\mathrm{n}=26)$ \\ Child Care/Day Care $(\mathrm{n}=14)$ \\ Preschool $(n=10)$ \\ Family Day Care $(\mathrm{n}=1)$ \\ Nurserv School $(\mathrm{n}=1)$}

Figure 1: Quorom Statement Flow Diagram. Identification of studies evaluating interventions aiming to improve children's nutrition delivered in early childcare settings.

'strong' where four of six key quality assessment criteria were rated as strong, with no weak ratings; 'moderate' if less than four criteria were rated strong and one criterion was rated weak; and 'weak' where two or more criteria were rated weak. Because of heterogeneity in study interventions, methodology and outcomes meta-analysis was not undertaken. Results are presented in narrative form. A study was classified as demonstrating 'intervention effectiveness' where there was at least one significant change (or improvement, when significance not tested or reported, $\mathrm{n}=3$ ) in outcomes measured.

\section{RESULTS}

\section{Study Description and Quality Assessment}

Table 1 summarizes the 26 intervention studies reviewed. Fifteen studies were published from 2010 onwards. Studies were primarily delivered in the child care/day care $(n=14)$ or preschool $(n=10)$ setting, with 
Table 1: Characteristics of Intervention Studies Conducted in Early Childhood Settings Aiming to Improve Children's Nutrition

\begin{tabular}{|c|c|c|c|c|c|}
\hline Reference & $\begin{array}{l}\text { Target } \\
\text { Participants }\end{array}$ & Design $^{3}$ & $\begin{array}{l}\text { Intervention } \\
\text { Duration; } \\
\text { Follow Up }\end{array}$ & Intervention Objective and Description & $\begin{array}{l}\text { Quality } \\
\text { Rating }\end{array}$ \\
\hline \multicolumn{6}{|c|}{ Child care / day care } \\
\hline $\begin{array}{l}\text { Cason et al. } \\
2001 \quad \text { [23]; } \\
\text { Southern } \\
\text { states, USA }\end{array}$ & $\begin{array}{l}\text { Children } \\
\text { ( } n=6102) \quad \text { and } \\
\text { educators }(n=46) \\
\text { from } 229 \text { centres }\end{array}$ & $\begin{array}{l}\text { Cohort } \\
\text { (1) Pre- Post- } \\
\text { Intervention }\end{array}$ & 24wk; NA & $\begin{array}{l}\text { To evaluate a multiple intelligences theory (MIT)- } \\
\text { based curriculum on children's fruit and vegetable } \\
\text { outcomes. } \\
\text { Educators: } 3 \times 6 \mathrm{hr} \text { training sessions on MIT, pre- } \\
\text { schooler nutrition, curriculum implementation. } \\
\text { Materials on MIT, activities for each intelligence, } \\
\text { lesson planning guide. } \\
\text { Children: } 12 \times 40 \text { min lessons on healthy snacking, } \\
\text { fruit and vegetable identification and the Food Guide } \\
\text { Pyramid }\end{array}$ & Moderate \\
\hline $\begin{array}{l}\text { Sangster et } \\
\text { al. } 2003 \text { [35]; } \\
\text { New South } \\
\text { Wales, } \\
\text { Australia }\end{array}$ & $\begin{array}{l}\text { Directors }(n=22) \\
\text { and } \\
(n=N R) \text { from } 15 \\
\text { centres }\end{array}$ & $\begin{array}{l}\text { Cohort } \\
\text { (1) Pre- Post- } \\
\text { Intervention }\end{array}$ & $2 y ; N A$ & $\begin{array}{l}\text { To improve the nutritional quality and safety of food } \\
\text { brought from home for children attending child care } \\
\text { centres. } \\
\text { Directors: Nutrition training and resources on healthy } \\
\text { food, food handling, communicating with parents, } \\
\text { nutrition policy } \\
\text { Parents: Information on healthy food, food handling, } \\
\text { centre policy on food brought from home }\end{array}$ & Weak \\
\hline $\begin{array}{l}\text { Matwiejczyk } \\
\text { et al. } 2007 \\
\text { [43]; South } \\
\text { Australia, } \\
\text { Australia }\end{array}$ & $\begin{array}{l}\text { Directors } \quad(n=40) \\
\text { and cooks }(n=47) \\
\text { from } 50 \text { centres }\end{array}$ & $\begin{array}{l}\text { Cohort } \\
\text { (1) Pre- Post- } \\
\text { Intervention }\end{array}$ & $30 \mathrm{~m} ; \mathrm{NR}$ & $\begin{array}{l}\text { To improve the nutrition provided to children } \\
\text { attending childcare centres via a nutrition award } \\
\text { initiative scheme 'Start Right Eat Right' } \\
\text { Cooks: 9-hour nutrition workshop, menu } \\
\text { improvement assignment } \\
\text { Directors: As for cooks, centre food policy review } \\
\text { All Staff: Food safety and hygiene training } \\
\text { Centre: Dietitian site visit and menu/policy review } \\
\text { support }\end{array}$ & Weak \\
\hline $\begin{array}{l}\text { Benjamin et } \\
\text { al. } 2007 \text { [45]; } \\
\text { North } \\
\text { Carolina, } \\
\text { USA }\end{array}$ & $\begin{array}{l}\text { Directors }(n=17) \\
\text { and staff }(n=51) \\
\text { from } 19 \text { centres }\end{array}$ & $\begin{array}{l}\text { Pilot CCT } \\
\text { (1) Intervention } \\
\text { (2) Control }\end{array}$ & $6 m ; 10 m$ & $\begin{array}{l}\text { To determine the feasibility and impact of a nutrition } \\
\text { and physical activity environmental intervention } \\
\text { (NAP SACC). } \\
\text { Directors and Staff: } 5 \text { step intervention 1) self- } \\
\text { assessment tool, 2) action planning, 3) continuing } \\
\text { education workshops, 4) technical assistance, 5) } \\
\text { repeat self-assessment tool }\end{array}$ & Weak \\
\hline $\begin{array}{l}\text { Benjamin et } \\
\text { al. } 2008 \text { [38]; } \\
\text { North } \\
\text { Carolina USA }\end{array}$ & $\begin{array}{l}\text { Health } \\
\text { consultants } \\
(n=49)\end{array}$ & $\begin{array}{l}\text { CCT } \\
\text { (1) Intervention (in } \\
\text { person) } \\
\text { (2) Intervention } \\
\text { (web access) } \\
\text { (3) Control }\end{array}$ & 10m; NA & $\begin{array}{l}\text { To compare web- versus in person- health } \\
\text { consultant training. } \\
\text { Health consultants trained to deliver NAP SACC } \\
\text { intervention via } \sim 3 \text { hours in-person training }(\sim 3 \\
\text { hours), or } 3 \text { week access to web site. }\end{array}$ & Weak \\
\hline $\begin{array}{l}\text { Ward et al. } \\
2008 \text { [37]; } \\
\text { North } \\
\text { Carolina, } \\
\text { USA }\end{array}$ & $\begin{array}{l}\text { Health } \\
\text { consultants } \\
(n=30)\end{array}$ & $\begin{array}{l}\text { CCT } \\
\text { Two Delivery } \\
\text { Methods } \\
\text { (1) Intervention } \\
\text { (in-person) } \\
\text { (2) Intervention } \\
\text { (web access) } \\
\text { Delayed- } \\
\text { intervention } \\
\text { control }\end{array}$ & $6 \mathrm{~m} ; \mathrm{NA}$ & $\begin{array}{l}\text { To test the NAP SACC intervention in promoting } \\
\text { healthy weight in young children. } \\
\text { Health consultants: in person or web-based NAP } \\
\text { SACC training } \\
\text { Staff: } 5 \text { step intervention delivered by health } \\
\text { consultants : 1) self-assessment tool, 2) action } \\
\text { planning, 3) continuing education workshops, 4) } \\
\text { technical assistance, } 5 \text { ) repeat self-assessment tool }\end{array}$ & Moderate \\
\hline
\end{tabular}


(Table 1). Continued.

\begin{tabular}{|c|c|c|c|c|c|}
\hline Reference & $\begin{array}{l}\text { Target } \\
\text { Participants }\end{array}$ & Design $^{3}$ & $\begin{array}{l}\text { Intervention } \\
\text { Duration; } \\
\text { Follow Up }\end{array}$ & Intervention Objective and Description & $\begin{array}{l}\text { Quality } \\
\text { Rating }\end{array}$ \\
\hline $\begin{array}{l}\text { Clark et al. } \\
2009 \text { [36]; } \\
\text { Colorado, } \\
\text { USA }\end{array}$ & Providers $(\mathrm{n}=38)$ & $\begin{array}{l}\text { Cohort } \\
\text { (1) Intervention } \\
\text { (2) Comparison }\end{array}$ & $3 m ; 6 m$ & $\begin{array}{l}\text { To evaluate the effectiveness of a website to } \\
\text { improve provider's infant feeding knowledge, } \\
\text { attitudes and behaviours. } \\
\text { Group } 1 \text { :Staff - InfaNET study website with } \\
\text { information on infant feeding: breast milk, formula, } \\
\text { solid foods } \\
\frac{\text { Group 2:Staff - health-related website specific to }}{\text { providers }}\end{array}$ & Moderate \\
\hline $\begin{array}{l}\text { Drummond et } \\
\text { al. } 2009 \text { [46]; } \\
\text { Arizona, USA }\end{array}$ & $\begin{array}{l}\text { Staff } \quad(n=337) \\
\text { from } 30 \text { centres }\end{array}$ & $\begin{array}{l}\text { Cohort } \\
\text { (1) Pre- Post- } \\
\text { Intervention }\end{array}$ & $9 \mathrm{~m} ; \mathrm{NA}$ & $\begin{array}{l}\text { To pilot a childhood obesity and diabetes prevention } \\
\text { intervention (NAP SACC) } \\
\text { Directors and Staff: Attend } 7 \text { workshops; 1) } \\
\text { childhood obesity, 2) self -assessment tool, 3) action } \\
\text { planning, 4) healthy eating for pre-schoolers, 4) } \\
\text { physical activity for pre-schoolers 5) personal health } \\
\text { - taking care of yourself 5) repeat self-assessment } \\
\text { tool }\end{array}$ & Weak \\
\hline $\begin{array}{l}\text { Sweitzer et } \\
\text { al. 2010 [32], } \\
\text { Briley et al. } \\
2012 \text { [31]; } \\
\text { Texas, USA }\end{array}$ & 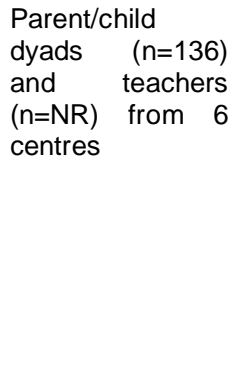 & $\begin{array}{l}\text { CCT } \\
\text { (1) Intervention } \\
\text { (2) Control }\end{array}$ & $11 w k ; N A$ & $\begin{array}{l}\text { To evaluate an intervention aimed at increasing } \\
\text { servings of fruits, vegetables and whole grains } \\
\text { brought from home } \\
\text { Parents: } 5 x \text { weekly handouts on nutrition, menu \& } \\
\text { recipe suggestions, goal-setting activities and social } \\
\text { references } \\
\text { Children: classroom nutrition activities and education } \\
\text { stations } \\
\text { Teachers: Training and information on all } \\
\text { intervention components to ensure high-fidelity } \\
\text { implementation }\end{array}$ & $\begin{array}{l}\text { Moderate } \\
\text { Moderate }\end{array}$ \\
\hline $\begin{array}{l}\text { Gosliner et al. } \\
2010 \quad \text { [42]; } \\
\text { California, } \\
\text { USA }\end{array}$ & $\begin{array}{l}\text { Providers } \\
(n=124) \text { from } 13 \\
\text { centres }\end{array}$ & $\begin{array}{l}\text { CCT } \\
\text { (1) Intervention } \\
\text { (2) Enhanced } \\
\text { Intervention }\end{array}$ & 10m; NA & $\begin{array}{l}\text { To evaluate the effect of a staff wellness program on } \\
\text { the child care nutrition and physical activity } \\
\text { environment. } \\
\text { Group } 1 \text { and } 2 \text { :Staff - training on children's health } \\
\text { and nutrition; set of nutrition and activity policies for } \\
\text { centre } \\
\text { Group } 1 \text { only (additional wellness program): Staff: } \\
\text { Additional } 1 \text { x day training workshop on adult } \\
\text { nutrition, } 9 \times \text { monthly staff walking group, follow up } \\
\text { support }\end{array}$ & Moderate \\
\hline $\begin{array}{l}\text { Herman et al. } \\
2012 \quad \text { [30]; } \\
\text { Pennsylvania, } \\
\text { Texas, } \\
\text { Arizona, } \\
\text { Rhode Island, } \\
\text { and New } \\
\text { York, USA }\end{array}$ & $\begin{array}{lr}\text { Staff } & (\mathrm{n}=496), \\
\text { parents } & (\mathrm{n}=438) \\
\text { and } & \text { children } \\
(\mathrm{n}=112) & \text { from 75 } \\
\text { Head } & \text { Start } \\
\text { centres } & \end{array}$ & $\begin{array}{l}\text { Cohort } \\
\text { (1) Pre- Post- } \\
\text { Intervention }\end{array}$ & $6 \mathrm{~m} ; \mathrm{NA}$ & $\begin{array}{l}\text { To pilot the 'Eat Healthy, Stay Active!" curriculum } \\
\text { promoting better nutrition and physical activity to } \\
\text { parents, staff, children. } \\
\text { Centre: site visits to ensure curriculum } \\
\text { implementation } \\
\text { Staff: A curriculum training session and } 3 \text { webinars. } \\
\text { Delivered program to parents. } \\
\text { Parents: Education sessions; My Pyramid food } \\
\text { groups, portion control, budget shopping. Nutrition } \\
\text { kills workshops. Family development support in goal } \\
\text { setting and reviewing changes. } \\
\text { Children: nutrition activity lessons e.g. cooking, fruit } \\
\text { and veg gardens, field trips. }\end{array}$ & Weak \\
\hline $\begin{array}{l}\text { Boyer et al. } \\
2012 \text { [41]; } \\
\text { Indiana, USA }\end{array}$ & $\begin{array}{l}\text { Children } \quad(n=28) \\
\text { from } 1 \text { centre }\end{array}$ & $\begin{array}{l}\text { Cross-over study } \\
\text { (1) Normal form } \\
\text { (2) Shaped form }\end{array}$ & 9 wk; NA & $\begin{array}{l}\text { To evaluate the effect of serving healthy, high-fibre } \\
\text { snack foods in normal or shaped form on } \\
\text { consumption } \\
\text { Children: regular snacks replaced with } 1 \text { of } 3 \text { high- } \\
\text { fibre, low-fat snacks 1) banana bread, 2) pancakes, } \\
\text { 3) turkey/cheese sandwiches presented in two } \\
\text { forms. }\end{array}$ & Moderate \\
\hline
\end{tabular}


(Table 1). Continued.

\begin{tabular}{|c|c|c|c|c|c|}
\hline Reference & $\begin{array}{l}\text { Target } \\
\text { Participants }\end{array}$ & Design $^{3}$ & $\begin{array}{l}\text { Intervention } \\
\text { Duration; } \\
\text { Follow Up }\end{array}$ & Intervention Objective and Description & $\begin{array}{l}\text { Quality } \\
\text { Rating }\end{array}$ \\
\hline $\begin{array}{l}\text { Witt et al. } \\
2012 \text { [26]; } \\
\text { Idaho, USA }\end{array}$ & $\begin{array}{l}\text { Children }(n=263) \text {, } \\
\text { parents and staff } \\
\text { from } 17 \text { centres }\end{array}$ & $\begin{array}{l}\mathrm{RCT} \\
\text { (1) Intervention } \\
\text { (2) Control }\end{array}$ & $6 w k ; 3 m$ & $\begin{array}{l}\text { To determine whether the Color Me Healthy }(C M H) \\
\text { program influences fruit and vegetable intake } \\
\text { Teachers: CMH "toolkit" including teacher's guide, } \\
\text { picture cards, posters, music, hand stamp, } \\
\text { reproducible parent newsletters. Materials } \\
\text { emphasise fruit and vegetables of different colours. } \\
\text { Children: received 12x'circle-time' lessons (15- } \\
30 \text { mins) and 6ximaginary trips e.g. use imagination } \\
\text { to eat fruit and veg. } \\
\text { Parents: newsletters on CMH content }\end{array}$ & Weak \\
\hline \multicolumn{6}{|l|}{ Preschool } \\
\hline $\begin{array}{l}\text { Gorelick et al. } \\
1985 \quad \text { [29]; } \\
\text { Unspecified, } \\
\text { USA }\end{array}$ & $\begin{array}{l}\text { Children }(n=187) \\
\text { from } 14 \text { schools }\end{array}$ & $\begin{array}{l}\text { CCT } \\
\text { (1) Intervention } \\
\text { with pretesting } \\
(2) \quad \text { Control } \\
\text { with pretesting } \\
(3) \quad \text { Intervention } \\
\text { without pretesting } \\
\text { Control without } \\
\text { pretesting }\end{array}$ & $6 w k ; N A$ & $\begin{array}{l}\text { To determine whether a nutrition education program } \\
\text { incorporating tasks appropriate to children's } \\
\text { cognitive level increases children's food knowledge } \\
\text { and nutrition. } \\
\text { Teachers: Trained to use program kit including an } \\
\text { assessment instrument, } 50 \text { x classroom activities, } \\
\text { materials to implement lessons, a recipe book, two } \\
\text { filmstrips. } \\
\text { Children: lessons on growing and preparing food, } \\
\text { developing cognitive skills, creative expression, } \\
\text { preparing nutritious snacks. }\end{array}$ & Weak \\
\hline $\begin{array}{l}\text { Hendy et al. } \\
2002 \quad \text { [40]; } \\
\text { Pennsylvania, } \\
\text { USA }\end{array}$ & $\begin{array}{l}\text { Children } \quad(n=38) \\
\text { from } 1 \text { preschool }\end{array}$ & $\begin{array}{l}\text { Cohort } \\
\text { (1) Pre- Post- } \\
\text { Intervention }\end{array}$ & $\begin{array}{l}\text { Immediate; } \\
1 \mathrm{~m}\end{array}$ & $\begin{array}{l}\text { To examine the effectiveness of trained peer models } \\
\text { to encourage food acceptance } \\
\text { Children: } 10 \text { min training by staff to be peer models } \\
\text { of food acceptance in exchange for small toy } \\
\text { reinforcements. Trained to say "These X taste good" } \\
\text { when on cue. }\end{array}$ & Weak \\
\hline $\begin{array}{l}\text { Williams et al. } \\
2002 \quad \text { [44], } \\
\text { New } \quad \text { York, } \\
\text { USA }\end{array}$ & $\begin{array}{l}\text { Food } \quad \text { service } \\
\text { staff, } \\
(1,296 \text { children })\end{array}$ & $\begin{array}{l}\text { CCT } \\
\text { (1) Intervention } \\
\text { enhanced } \\
\text { (2) Intervention } \\
\text { (3) Control }\end{array}$ & $\begin{array}{l}\sim 6 \text { months; } \\
\text { NA }\end{array}$ & $\begin{array}{l}\text { To reduce the saturated fat content of preschool } \\
\text { meals through a food service improvement } \\
\text { intervention } \\
\text { Group } 1 \text { (FS/NU): Cooks }-1 \text { x day food service } \\
\text { training } 2.5 y \text { of monthly site visits. Parents -nutrition } \\
\text { pamphlets and quarterly nutrition education } \\
\text { sessions.Teachers -skills-based nutrition curriculum } \\
\text { training. } \\
\text { Group } 2 \text { (FS only): Cooks - As for Group } 1 . \\
\text { Teachers- trained in 'general health' curriculum. } \\
\text { Group } 3 \text { (control): Teachers- trained in 'general } \\
\text { health' curriculum. }\end{array}$ & Weak \\
\hline $\begin{array}{l}\text { Fitzgibbon et } \\
\text { al. } 2005 \text { [27]; } \\
\text { Chicago, } \\
\text { USA (Head } \\
\text { Start) }\end{array}$ & $\begin{array}{l}\text { Children }(\mathrm{n}=409) \\
\text { from } \\
\text { 'black'preschools }\end{array}$ & $\begin{array}{l}\text { RCT } \\
\text { (1) Intervention } \\
\text { (2) Control }\end{array}$ & $14 w k ; 1 y, 2 y$ & $\begin{array}{l}\text { To determine whether a diet and activity intervention } \\
\text { reduces gains in BMI } \\
\text { Children: 14-wk (3xweekly; 20min nutrition, } 20 \text { min } \\
\text { PA) diet and activity curriculum delivered by trained } \\
\text { educators. } \\
\text { Parents: weekly newsletters and homework } \\
\text { assignments reinforcing the curriculum }\end{array}$ & Moderate \\
\hline $\begin{array}{l}\text { Fitzgibbon et } \\
\text { al. } 2006 \text { [28]; } \\
\text { Chicago, } \\
\text { USA (Head } \\
\text { Start) }\end{array}$ & $\begin{array}{l}\text { Children ( } n=401) \\
\text { from } 12 \text { 'Latino' } \\
\text { preschools }\end{array}$ & $\begin{array}{l}\text { RCT } \\
\text { (1) Intervention } \\
\text { (2) Control }\end{array}$ & $14 w k ; 1 y, 2 y$ & As above - Fitzgibbon et al. 2005 (27) & Moderate \\
\hline $\begin{array}{l}\text { Hardy et al. } \\
2010 \quad \text { [48]; } \\
\text { Sydney, } \\
\text { Australia }\end{array}$ & $\begin{array}{lr}\text { Staff } \quad(n=73 \\
\text { baseline, } \quad n=65 \\
\text { FU) from } \quad 29 \\
\text { preschools }\end{array}$ & $\begin{array}{l}\text { RCT } \\
\text { (1) Intervention } \\
\text { (2) Control }\end{array}$ & $6 \mathrm{~m} ; \mathrm{NA}$ & $\begin{array}{l}\text { To evaluate 'Munch and Move': a low-intensity, early } \\
\text { childhood professional development program } \\
\text { Staff: 1d workshop on healthy eating, food-based } \\
\text { education activities, activity, nutrition and } \\
\text { activitypolicies } \\
\text { Preschools: resources; contact with health } \\
\text { promotion professionals to support program delivery. }\end{array}$ & Weak \\
\hline
\end{tabular}


(Table 1). Continued.

\begin{tabular}{|c|c|c|c|c|c|}
\hline Reference & $\begin{array}{l}\text { Target } \\
\text { Participants }\end{array}$ & Design $^{3}$ & $\begin{array}{l}\text { Intervention } \\
\text { Duration; } \\
\text { Follow Up }\end{array}$ & Intervention Objective and Description & $\begin{array}{l}\text { Quality } \\
\text { Rating }\end{array}$ \\
\hline $\begin{array}{l}\text { De Bock et } \\
\text { al. } 2010 \text { [24]; } \\
\text { Baden- } \\
\text { Wurttemberg, } \\
\text { Germany }\end{array}$ & $\begin{array}{l}\text { Parent/child } \\
\text { dyads } \quad(n=348) \\
\text { from } \\
\text { preschools }\end{array}$ & $\begin{array}{l}\text { Cross-over } \\
\text { Cluster-RCT } \\
\text { (1) Intervention } \\
\text { (2) Control }\end{array}$ & $6 m ; N A$ & $\begin{array}{l}\text { To assess the short-term impact of a nutritional } \\
\text { intervention aimed at reducing childhood overweight } \\
\text { Fifteen } 2 \mathrm{~h} \text { sessions } 1 \times \mathrm{xk} \text { over } 6 \mathrm{~m} \text {; ten target } \\
\text { children, } 5 \text { target parents and children or parents } \\
\text { exclusively. Themes were: } \\
\text { Children: curriculum education and activities focused } \\
\text { on fruit and vegetables; water } \\
\text { Parents: balanced nutrition; children's eating } \\
\text { behaviour; sharing experiences } \\
\text { Parents and children: preparing fruit and vegetable } \\
\text { snacks } \\
\text { Fathers and children: fathers baking with their } \\
\text { children }\end{array}$ & Weak \\
\hline $\begin{array}{l}\text { Sharma et al. } \\
2011 \quad \text { [25]; } \\
\text { Texas, USA } \\
\text { (Head Start) }\end{array}$ & $\begin{array}{l}\text { Children }(n=75) \text {, } \\
\text { their parents; } 9 \\
\text { teachers from } 2 \\
\text { centres }\end{array}$ & $\begin{array}{l}\text { Pilot cohort } \\
\text { (1) Pre-Post- } \\
\text { Intervention }\end{array}$ & $6 w k ; N A$ & $\begin{array}{l}\text { To pilot test the CATCH Early Childhood (CEC) } \\
\text { intervention } \\
\text { Children: 9xnutrition lessons promoting healthful } \\
\text { eating habits; } \\
\text { Parents: 9xtip-sheets to modify home nutrition } \\
\text { environment } \\
\text { Teachers: Trained on implementing all CEC } \\
\text { components }\end{array}$ & Moderate \\
\hline $\begin{array}{l}\text { Zask et al. } \\
2012 \text { [33]; } \\
\text { NSW, } \\
\text { Australia }\end{array}$ & $\begin{array}{l}\text { Staff, parents and } \\
\text { children }(n=560) \\
\text { from } \\
\text { preschools }\end{array}$ & $\begin{array}{l}\text { Cross-over RCT } \\
\text { quasi- } \\
\text { experimental } \\
\text { design } \\
\text { (1) Intervention } \\
\text { (2) Control }\end{array}$ & $9 \mathrm{~m} ; \mathrm{NA}$ & $\begin{array}{l}\text { To increase fruit and vegetable serves, and reduce } \\
\text { amount of unhealthy snack items, bought to and } \\
\text { consumed in preschools } \\
\text { Staff: Nutrition policy review. 'Family Feud Food } \\
\text { DVD. Manual for including nutrition messages in art, } \\
\text { drama, role-play, music and stories; role modelling; } \\
\text { positive reinforcement } \\
\text { Parents: Interactive workshops on positive parenting } \\
\text { and healthy eating and feeding 'fussy eaters'. } \\
\text { Children: consistent messages on 'sometimes' and } \\
\text { 'everyday' foods; nutrition activities, and taste testing } \\
\text { fruit and vegetables. }\end{array}$ & Weak \\
\hline $\begin{array}{l}\text { Korenman et } \\
\text { al. } 2013 \text { [47]; } \\
9 \text { states, USA }\end{array}$ & 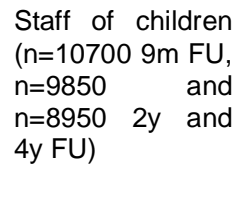 & $\begin{array}{l}\text { Cohort } \\
\text { (1) Intervention } \\
\text { (2) Comparison }\end{array}$ & $9 m ; 2 y, 4 y$ & $\begin{array}{l}\text { To determine associations between CACFP } \\
\text { participation and children's food consumption, } \\
\text { weight and family food security } \\
\text { Centres: reimbursed for meals and snacks served to } \\
\text { children on a per-meal, per-child basis according to } \\
\text { child's family income }\end{array}$ & Moderate \\
\hline \multicolumn{6}{|c|}{ Nursery school } \\
\hline $\begin{array}{l}\text { Horne et al. } \\
2011 \text { [39]; } \\
\text { Bangor, } \\
\text { Gwynedd, UK }\end{array}$ & $\begin{array}{l}\text { Children } \quad(n=20) \\
\text { from } 1 \text { nursery } \\
\text { school }\end{array}$ & $\begin{array}{l}\text { Cohort } \\
\text { (1) Pre- Post- } \\
\text { Intervention }\end{array}$ & $124 d ; 6 m$ & $\begin{array}{l}\text { To determine whether a modelling and rewards } \\
\text { intervention increases children's fruit and vegetable } \\
\text { consumption } \\
\text { Children: watched } 1 \times 5.5 \text { min videos - two animate } \\
\text { characters model eating a fruit and vegetable and } \\
\text { urged children to "eat them up to be big and strong"; } \\
\text { supporting letters read to children by staff; received } \\
\text { rewards (e.g. sticker, badge) for eating target food. }\end{array}$ & Weak \\
\hline \multicolumn{6}{|c|}{ Family Day Care } \\
\hline $\begin{array}{l}\text { Bravo et al. } \\
2008[34] \\
\text { New South } \\
\text { Wales, } \\
\text { Australia }\end{array}$ & $\begin{array}{l}\text { Staff (pre-post, } \\
\mathrm{n}=18-22), \quad \text { carers } \\
\text { (pre-post, } 102- \\
104), \text { and } \\
\text { parents } \\
\text { from } 7 \text { FDC } \\
\text { schemes }\end{array}$ & $\begin{array}{l}\text { Cohort } \\
\text { (interrupted time } \\
\text { series) } \\
\text { Pre-Post- } \\
\text { Intervention }\end{array}$ & $2-5 y ; N A$ & $\begin{array}{l}\text { To pilot a staff knowledge and skills nutrition and } \\
\text { food safety intervention aimed at improving nutrition } \\
\text { provided to children. } \\
\text { Staff and carers: } 1 \text { x workshop, support, nutrition } \\
\text { information kit including communicating/negotiating } \\
\text { with parents, food policy and safety. } \\
\text { Parents: Nutrition Information Kit }\end{array}$ & Moderate \\
\hline
\end{tabular}

Abbreviations: BMI: Body Mass Index; CACFP: Child and adult care food program; CCT: controlled clinical trial; d: day; FS: food service; FU: follow up; m: months NA: not applicable; NAPSACC: Nutrition and Physical Activity Self-Assessment for Child Care; NU: nutrition education; PA: Physical Activity; USA: United States of America; wk: weeks; y: years. 
one study set in Family Day Care and another in nursery schools. The majority $(n=19)$ of studies were conducted in the USA, with the remaining conducted in Australia $(n=5)$, Germany $(n=1)$ and the UK $(n=1)$. Six studies were randomized controlled trials, eight were clinical controlled trials and twelve were pre-post cohort studies with no control or comparison group. In assessing the methodological quality of included studies, 14 were rated as weak and 12 as moderate quality. No studies were rated as strong methodological quality. Studies performed most poorly on the criteria relating to potential selection bias, management of confounders and validity of data collection methods. Intervention exposure ranged from 1 hour to 2-5 years. Eight interventions were $<3$ months in duration, six lasted $\sim 6$ months and eight were $>6$ months in duration (Table 1).

The majority of studies $(n=20)$ targeted staff (directors, cooks, educators, floor staff or health consultants) as part of the intervention (Table 2). Over half $(n=15)$ of the studies targeted children directly as part of the intervention, and only 12 included a parental component. Less than half of studies $(n=12)$ targeted more than one participant group (staff, parents, children) i.e. multi-target interventions [23-35]. Group training sessions $(n=20)$, written materials $(n=17)$ and nutrition curriculum for children $(n=11)$ were the most utilised modes of intervention delivery (Table 2). Three studies used a website to deliver the intervention [3638], one used a video [39] and two trained children to be role models with their eating $[39,40]$. Most $(n=21)$ interventions focused on 'healthy' eating, either in general or specifically in relation to dietary guidelines, such as infant feeding $(n=1)$ [36], fruit and vegetables $(n=7)[23,24,26,31-33,39]$ or whole grains or fibre intake $(n=3)[31,32,41]$. Nutrition policies $(n=5)[33-35$, $42,43]$, enhancing nutrition skills $(n=5)[24,29,30,43$, $44]$, food service modification $(n=2)[43,44]$ and role modelling $(n=2)[39,40]$ were other nutrition strategies utilised. All but five [24, 36, 40, 45, 46] studies used a combination of intervention delivery modes. Nine interventions, detailed in eleven studies [23-25, 27, 28, 30 , 36-38, 40, 45], reported being based on a behaviour change theory theoretical framework. The most common were social cognitive theory $[25,27,28$, $37,38,40,45]$, social-ecological framework [37, 38, 45] and social learning theory $[24,30,36]$.

\section{Intervention Content by Participant Group}

\section{Staff Component}

Nutrition training for directors, cooks, and/or floor staff included content such as children's nutritional requirements [23, 42, 43], healthy foods [34, 35, 42, $43]$, handling food safely [34, 35, 43], nutrition policy [33-35, 43], parent communication skills [34, 35], food service/menu modification $[43,44]$ and nutrition curriculum [23, 25, 26, 29-33, 44]. One study trained staff in a wellness program, aiming to empower them to improve their own health [42]. Several studies reported on an intervention which involved training of health consultants on nutrition and physical activity for children and adults and providing consultation to child care centres [37, 38], followed by intervention implementation in centres [37, 45, 46]. Similarly, the "Start Right-Eat Right" intervention employed Dieticians to train child care centre directors and cooks on children's nutrition, menus and policies, and provide consultation and support until practices and documents met award criteria [43].

\section{Parent Component}

Of the twelve studies that engaged parents of children attending ECS, three aimed to improve food bought from home $[31,32,35]$ whilst the remaining studies did so primarily as an effective means to modify children's behaviour. Parents were provided with written information on a variety of topics including children's healthy food choices, menu and recipe suggestions, food safety, special diets, healthy eating activities and food brought from home. Three studies engaged parents through delivering parent education workshops on child nutrition, positive food parenting or nutrition skills such as budgeting [24, 30, 33].

\section{Child Component}

Studies targeting children as part of the intervention usually did so through a structured nutrition curriculum delivered by trained educators [27, 28] or staff [23-30, 33]. Some focused on practical activities, including growing fruit and vegetables, cooking lessons, arts and crafts, games, and field trips to farmers markets and grocery stores [23, 24, 32, 33]. One study evaluated the effect of serving different shaped food on children's consumption [41] and two investigated the use of children as peer role models $[39,40]$.

\section{Intervention Outcomes}

Study outcomes are summarised in Table 2. Nearly all studies $(n=23)$ demonstrated intervention effectiveness. Of the three studies where results did not support intervention effectiveness, all were rated moderate quality $[25,28,41]$. 
Table 2: Impact of Early Childcare Setting Interventions on Nutrition Outcome Measures by Participant Group

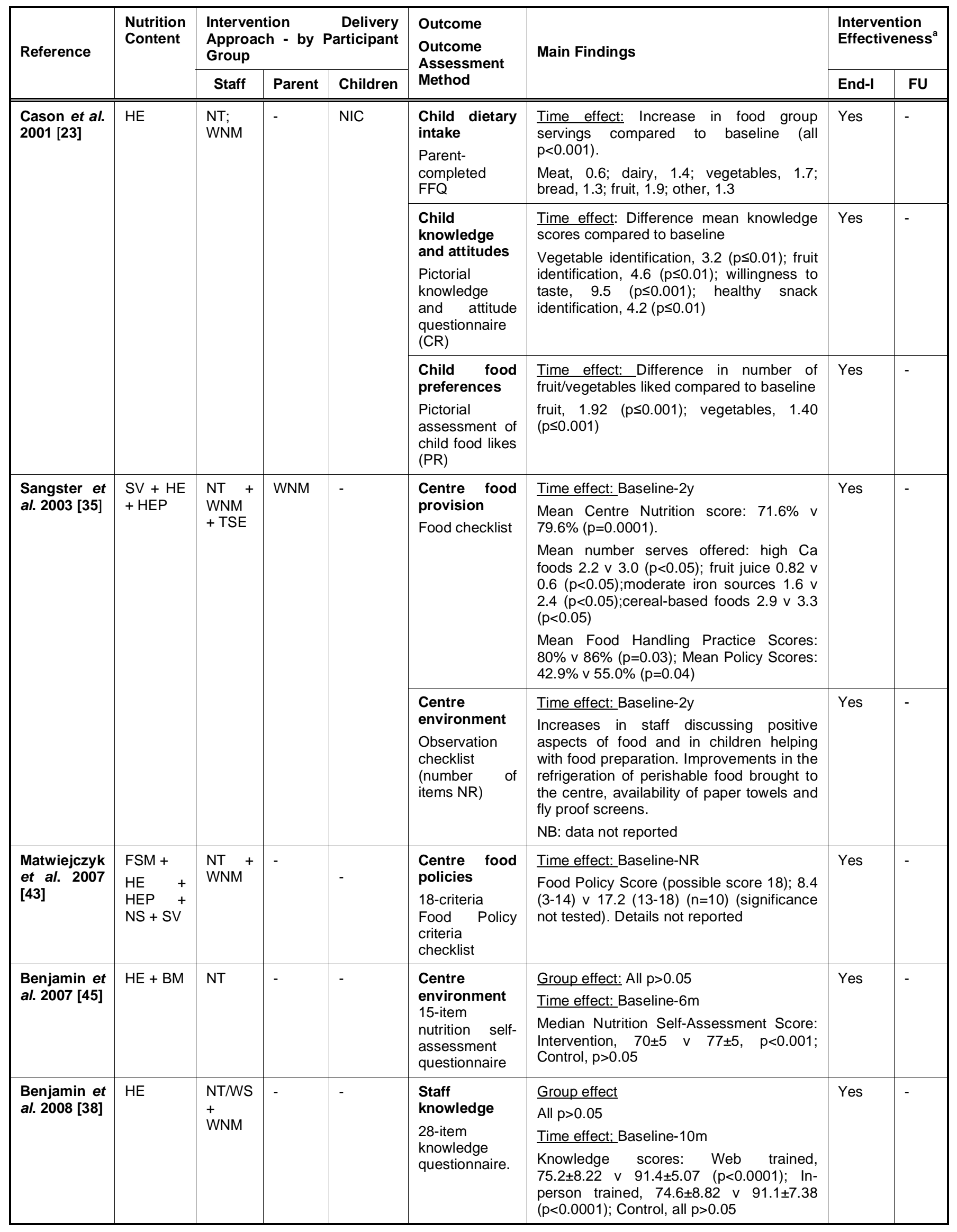


(Table 2). Continued.

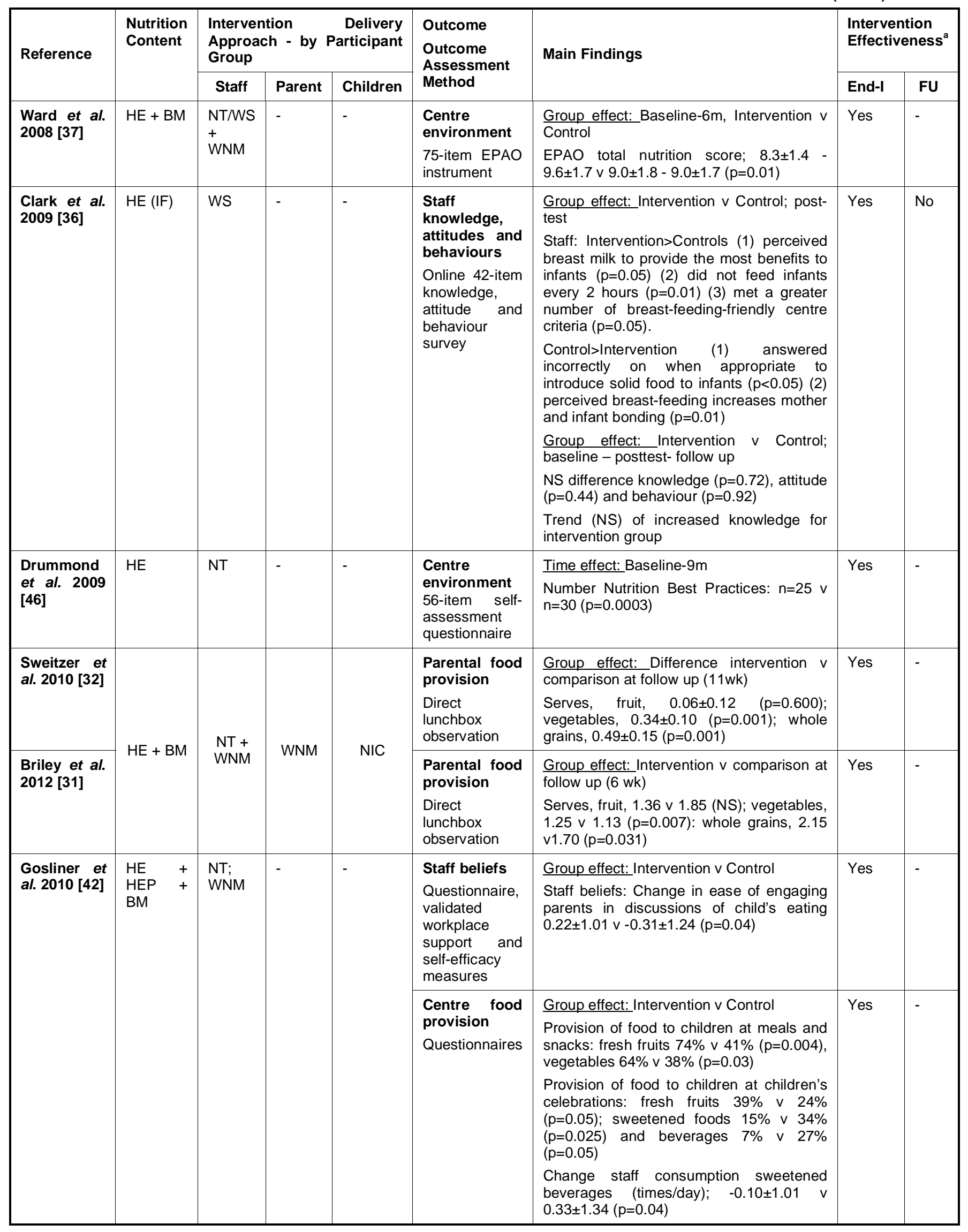


(Table 2). Continued.

\begin{tabular}{|c|c|c|c|c|c|c|c|c|}
\hline \multirow[t]{2}{*}{ Reference } & \multirow[t]{2}{*}{$\begin{array}{l}\text { Nutrition } \\
\text { Content }\end{array}$} & \multicolumn{3}{|c|}{ 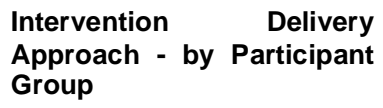 } & \multirow{2}{*}{$\begin{array}{l}\text { Outcome } \\
\text { Outcome } \\
\text { Assessment } \\
\text { Method }\end{array}$} & \multirow[t]{2}{*}{ Main Findings } & \multicolumn{2}{|c|}{$\begin{array}{l}\text { Intervention } \\
\text { Effectiveness }^{a}\end{array}$} \\
\hline & & Staff & Parent & Children & & & End-I & FU \\
\hline \multirow[t]{2}{*}{$\begin{array}{l}\text { Herman et } \\
\text { al. } 2012 \text { [30] }\end{array}$} & \multirow[t]{2}{*}{$\begin{array}{l}\mathrm{NS}+\mathrm{SV} \\
+\mathrm{BM}\end{array}$} & \multirow[t]{2}{*}{ NT } & \multirow[t]{2}{*}{$\begin{array}{l}\mathrm{NT} \\
\mathrm{BM}\end{array}+$} & \multirow[t]{2}{*}{$\mathrm{NIC}$} & $\begin{array}{l}\text { Parental } \\
\text { knowledge } \\
\text { Diet \& PA } \\
\text { knowledge } \\
\text { and behaviour } \\
\text { questionnaire }\end{array}$ & $\begin{array}{l}\text { Time effect: } \\
\text { Knowledge (\% correct) [mean (SD)], } 93.7 \\
(7.0)-94.6(5.8) \text { : difference } 0.9, p \leq 0.001 \\
\text { Diet behaviours (scores scaled to 100) } \\
\text { [mean (SD)], } 74.0(9.8)-76.2(9.3), 2.2 \\
\text { (p } \leq 0.001) \\
\text { NB: results for parents and staff combined }\end{array}$ & Yes & - \\
\hline & & & & & $\begin{array}{l}\text { Staff } \\
\text { knowledge } \\
\text { Diet \& PA } \\
\text { knowledge } \\
\text { and behaviour } \\
\text { questionnaire }\end{array}$ & $\begin{array}{l}\text { Time effect: } \\
\text { Knowledge (\% correct), }-0.9, p \leq 0.001 \\
\text { Diet behaviours (scores scaled to } 100), 2.2 \\
(p \leq 0.001) \\
\text { NB: results for parents and staff combined }\end{array}$ & Yes & - \\
\hline $\begin{array}{l}\text { Boyer et al. } \\
2012 \text { [41] }\end{array}$ & MFP & - & - & DSS & $\begin{array}{l}\text { Child dietary } \\
\text { intake } \\
\text { Plate wastage } \\
\text { method }\end{array}$ & $\begin{array}{l}\text { Group effect; } \\
\text { No difference in average snack } \\
\text { consumption between shaped and normal } \\
\text { form snacks }(p=0.16)\end{array}$ & No & - \\
\hline $\begin{array}{l}\text { Witt et al. } \\
2012 \text { [26] }\end{array}$ & $\mathrm{HE}$ & WNM & WNM & $\mathrm{NIC}$ & $\begin{array}{l}\text { Children's } \\
\text { dietary intake } \\
\text { Plate wastage } \\
\text { method }\end{array}$ & 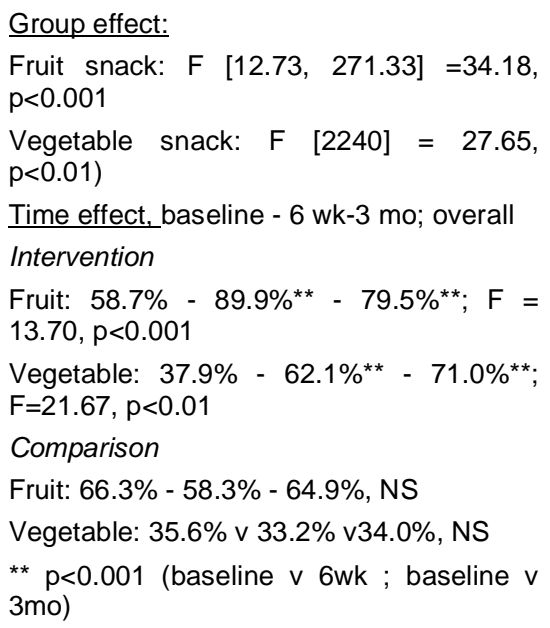 & Yes & Yes \\
\hline $\begin{array}{l}\text { Gorelick et } \\
\text { al. } 1985 \text { [29] }\end{array}$ & $\mathrm{HE}+\mathrm{NS}$ & $\begin{array}{l}\text { NT }+ \\
\text { WNM } \\
+ \text { TSE }\end{array}$ & - & $\mathrm{NIC}$ & $\begin{array}{l}\text { Child } \\
\text { knowledge } \\
\text { 7-part } \\
\text { questionnaire } \\
\text { measuring } \\
\text { how well } \\
\text { children can } \\
\text { manipulate } \\
\text { and respond to } \\
\text { various foods } \\
\text { in a series of } \\
\text { game-like } \\
\text { tasks }\end{array}$ & $\begin{array}{l}\text { Group effect: Group } 1 \text { and } 3 \text { (experimental } \\
\text { groups) v groups } 2 \text { and } 4 \text { (control); } \\
\text { Overall posttest scores ( } p<0.001) \\
\text { Scores on 'identification of foods' } \\
\text { (p<0.001) } \\
\text { Scores on 'handwashing' }(p<0.05) \\
\text { Group } 1 \text { v group 3; NS }\end{array}$ & Yes & - \\
\hline $\begin{array}{l}\text { Hendy et al. } \\
2002 \text { [40] }\end{array}$ & FRR & - & - & $\mathrm{RMT}$ & $\begin{array}{l}\text { Child dietary } \\
\text { intake } \\
\text { Trained } \\
\text { researcher } \\
\text { observed food } \\
\text { bites. }\end{array}$ & $\begin{array}{l}\text { Time effect: } \\
\text { Food acceptance; An increase in food } \\
\text { bites observed at intervention delivery (girl } \\
\text { models only, } p=0.01) \text { but not } 1 \text {-month later } \\
(p=0.56)\end{array}$ & Yes & No \\
\hline & & & & & $\begin{array}{l}\text { Child food } \\
\text { preference } \\
\text { Food } \\
\text { preference } \\
\text { rating }\end{array}$ & $\begin{array}{l}\text { Time effect: } \\
\text { Food preference; No effects observed on } \\
\text { food preference ratings }(p=0.73)\end{array}$ & - & No \\
\hline
\end{tabular}




\begin{tabular}{|c|c|c|c|c|c|c|c|c|}
\hline Reference & $\begin{array}{l}\text { Nutrition } \\
\text { Content }\end{array}$ & \multicolumn{3}{|c|}{ 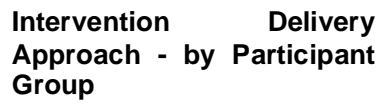 } & $\begin{array}{l}\text { Outcome } \\
\text { Outcome } \\
\text { Assessment } \\
\text { Method }\end{array}$ & Main Findings & \multicolumn{2}{|c|}{$\begin{array}{l}\text { Intervention } \\
\text { Effectiveness }^{a}\end{array}$} \\
\hline \multirow[t]{3}{*}{$\begin{array}{l}\text { Williams et } \\
\text { al. } 2002 \text { [44] }\end{array}$} & \multirow[t]{3}{*}{$\begin{array}{l}\mathrm{HE}+\mathrm{NS} \\
+\mathrm{FSM}\end{array}$} & \multirow[t]{3}{*}{ NT } & \multirow[t]{3}{*}{$\begin{array}{l}\text { NT }+ \\
\text { WNM }^{+}\end{array}$} & \multirow[t]{3}{*}{-} & $\begin{array}{l}\text { Child dietary } \\
\text { intake } \\
\text { Direction } \\
\text { observation } \\
\text { school meals } \\
\text { (plate wastage } \\
\text { method) }\end{array}$ & 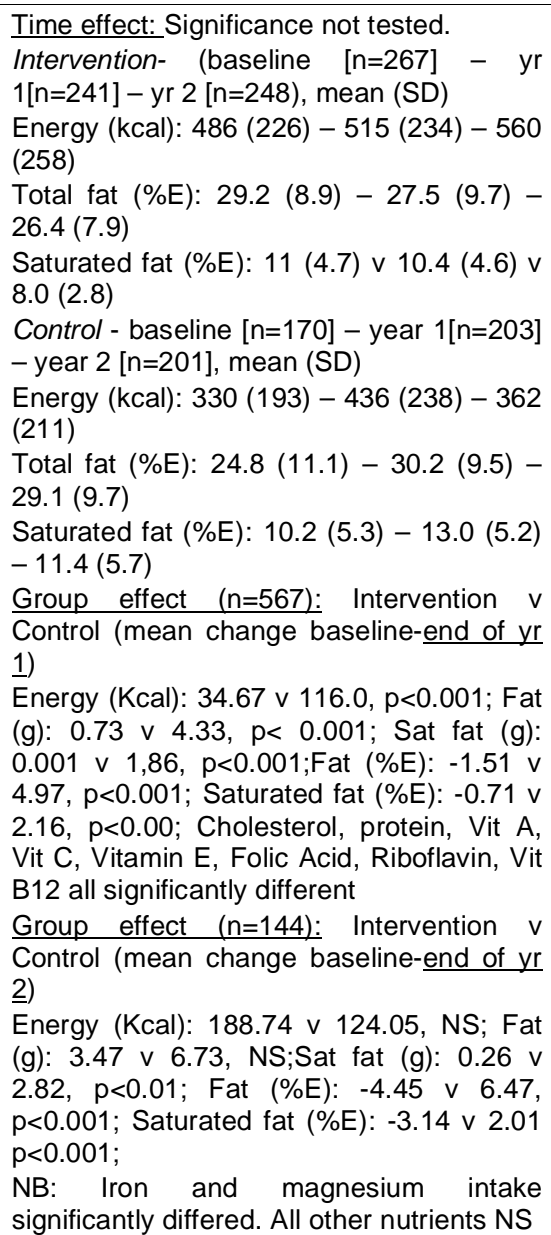 & Yes & Yes \\
\hline & & & & & $\begin{array}{l}\text { Child dietary } \\
\text { intake } \\
24 \mathrm{hr} \text { recalls } \\
\text { (direct } \\
\text { observation } \\
\text { during school, } \\
\text { parental from } \\
\text { reports fome) } \\
\text { home) }\end{array}$ & $\begin{array}{l}\text { Group effect: Intervention } \vee \text { Control } \\
\text { (baseline-end yr 1-end yr 2), mean (SD) } \\
\text { Saturated fat }(\% \mathrm{E}): 12(3.7)-11.6(3.5)- \\
10.9(3.1) \vee 12.4(3.2)-12.4(3.6)-12.4 \\
\text { (3.5) } \\
\text { NB: Significance not tested. Macro/micro- } \\
\text { nutrients reported, } \mathrm{n}=18 \text {. Intervention } \\
\text { aimed primarily at decreasing saturated } \\
\text { fat. }\end{array}$ & Yes & Yes \\
\hline & & & & & $\begin{array}{l}\text { Centre food } \\
\text { provision } \\
\text { 5-day menu } \\
\text { analysis }\end{array}$ & 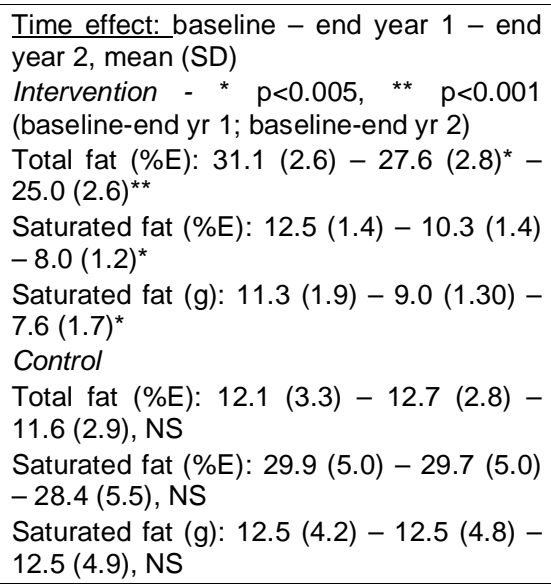 & Yes & Yes \\
\hline
\end{tabular}


(Table 2). Continued.

\begin{tabular}{|c|c|c|c|c|c|c|c|c|}
\hline \multirow[t]{2}{*}{ Reference } & \multirow[t]{2}{*}{$\begin{array}{l}\text { Nutrition } \\
\text { Content }\end{array}$} & \multicolumn{3}{|c|}{$\begin{array}{l}\text { Intervention } \quad \text { Delivery } \\
\text { Approach - by Participant } \\
\text { Group }\end{array}$} & \multirow{2}{*}{$\begin{array}{l}\text { Outcome } \\
\text { Outcome } \\
\text { Assessment } \\
\text { Method }\end{array}$} & \multirow[t]{2}{*}{ Main Findings } & \multicolumn{2}{|c|}{$\begin{array}{l}\text { Intervention } \\
\text { Effectiveness }^{a}\end{array}$} \\
\hline & & Staff & Parent & Children & & & End-I & $\mathbf{F U}$ \\
\hline $\begin{array}{l}\text { Fitzgibbon } \\
\text { et al. } 2005 \\
{[27]}\end{array}$ & \multirow{3}{*}{$\mathrm{HE}$} & \multirow{3}{*}{-} & \multirow{3}{*}{$\begin{array}{l}\mathrm{NT}+ \\
\text { WNM }\end{array}$} & \multirow{3}{*}{$\mathrm{NIC}$} & $\begin{array}{l}\text { Child dietary } \\
\text { intake } \\
\text { Parent- recall } \\
\text { of previous } \\
24 \mathrm{~h} \text { food } \\
\text { intake (full or } \\
\text { partial) }\end{array}$ & $\begin{array}{l}\text { Group effect: Intervention v Control (mean } \\
\text { difference (SE): post intervention; year } 1 \\
\text { FU; year } 2 \text { FU } \\
\text { Post-intervention } \\
\text { Total fat }(\% E):-1.16(-3.02 \text { to } 0.71) \mathrm{NS} \text {; } \\
1.46(-3.60 \text { to } 0.67) \mathrm{NS} \text { : } 0.58(-2.00 \text { to } \\
3.16) \mathrm{NS} \\
\text { Saturated fat }(\% \mathrm{E}):-0.65(-1.45 \text { to } 0.14) \\
\mathrm{NS} ;-1.15(-1.74 \text { to }-0.56) \mathrm{p}=0.002 ; 0.27(- \\
0.89 \text { to } 1.43) \mathrm{NS} \\
\text { Fibre }(\mathrm{g} / 1000 \mathrm{kcal}): 0.31(-0.87 \text { to } 1.49) \mathrm{NS} \text {; } \\
0.04(-0.96 \text { to } 1.04) \mathrm{NS} ;-0.58(-1.61 \text { to } \\
0.44) \mathrm{NS}\end{array}$ & No & Yes \\
\hline \multirow[t]{2}{*}{$\begin{array}{l}\text { Fitzgibbon } \\
\text { et al. } 2006 \\
\text { [28] }\end{array}$} & & & & & $\begin{array}{l}\text { Child dietary } \\
\text { intake } \\
\text { Parent- recall } \\
\text { of previous } \\
24 \mathrm{~h} \text { food } \\
\text { intake (full or } \\
\text { partial) }\end{array}$ & $\begin{array}{l}\text { Group effect: Intervention v Control mean } \\
\text { difference (SE): post intervention; year } 2 \\
\text { FU } \\
\text { Post-intervention } \\
\text { Total fat (\%kcal): } 0.02(-1.95 \text { to } 1.99) \mathrm{NS} ;- \\
0.25(-2.32 \text { to } 1.83) \mathrm{NS} \\
\text { Saturated fat (\%kcal): } 0.07(-1.75 \text { to } 0.89) \\
\text { NS; }-0.14(-0.76 \text { to } 0.49) \mathrm{NS} \\
\text { Fibre (g/1000kcal): } 0.80(-0.26 \text { to } 1.85) \mathrm{NS} \text {; } \\
-0.47(-2.07 \text { to } 1.13) \mathrm{NS}\end{array}$ & No & No \\
\hline & & & & & $\begin{array}{l}\text { Child weight } \\
\text { status } \\
\text { Trained } \\
\text { personnel. } \\
2000 \quad \text { CDC } \\
\text { growth charts }\end{array}$ & $\begin{array}{l}\text { Group effect: intervention v control (mean } \\
\text { change) } \\
\text { 14wk: Adjusted BMI z score, } 0.01(-0.19 \text { to } \\
0.20), p=0.94 \\
\text { Year 1: Adjusted BMI z score, }-0.11(-0.34 \\
\text { to } 0.11) p=0.29 \\
\text { Year 2: Adjusted BMl z score, }-0.15(-0.38 \\
\text { to } 0.09) p=0.19\end{array}$ & No & $\mathrm{N}$ \\
\hline $\begin{array}{l}\text { Hardy et al. } \\
2010 \text { [48] }\end{array}$ & $\mathrm{HE}+\mathrm{NS}$ & $\begin{array}{l}\text { NT } \\
\text { WNM } \\
+ \text { PC }\end{array}$ & - & - & $\begin{array}{l}\text { Parental food } \\
\text { provision } \\
\text { Direct } \\
\text { lunchbox } \\
\text { observation }\end{array}$ & $\begin{array}{l}\text { Group effect: Intervention v comparison at } \\
\text { follow up } \\
\text { Serves, fruit }(-0.05[-0.36,0.26], p=0.75) \text {, } \\
\text { vegetables }(0.12[-0.005,0.30], p=0.16) \text {, } \\
\text { snacks }(0.06 \quad[-0.34,0.46], \quad p=0.75) \text {, } \\
\text { sweetened drinks }(-0.13 \quad[-0.27,0.002] \text {, } \\
p=0.05) \text {, total extra food/drinks }(-0.6[-0.45 \text {, } \\
0.33], p=0.76)\end{array}$ & Yes & - \\
\hline \multirow[t]{2}{*}{$\begin{array}{l}\text { De Bock et } \\
\text { al. } 2012 \text { [24] }\end{array}$} & \multirow[t]{2}{*}{$\mathrm{HE}+\mathrm{NS}$} & \multirow[t]{2}{*}{-} & \multirow[t]{2}{*}{ NT } & \multirow[t]{2}{*}{ NIC } & $\begin{array}{l}\text { Child weight } \\
\text { status } \\
\text { Trained } \\
\text { personnel. }\end{array}$ & $\begin{array}{l}\text { Group effect:Intervention } \\
\text { No significant changes in BMI, WTHR, or } \\
\text { skinfold sum }\end{array}$ & No & - \\
\hline & & & & & $\begin{array}{l}\text { Child dietary } \\
\text { intake } \\
\text { Semi- } \\
\text { quantitative } \\
\text { parent- } \\
\text { completed } \\
\text { eating } \\
\text { behaviour } \\
\text { questionnaire }\end{array}$ & $\begin{array}{l}\text { Group effect:Intervention } \\
\text { Fruit, increase } 0.23(p=0.001) \text {; vegetables, } \\
\text { increase } 0.15 \quad(p=0.027) \text {. NS water or } \\
\text { sugared drinks. *change of } 1 \text { on the 6- } \\
\text { point ordinal scale approximates to one } \\
\text { portion difference (size of child's hand) }\end{array}$ & Yes & - \\
\hline $\begin{array}{l}\text { Sharma et } \\
\text { al. } 2011 \text { [25] }\end{array}$ & $\mathrm{HE}$ & NT & WNM & NIC & $\begin{array}{l}\text { Child dietary } \\
\text { intake } \\
\text { Plate wastage } \\
\text { method }\end{array}$ & $\begin{array}{l}\text { Time effect: } \\
\text { Fruit serves, } 0.6 \pm 0.4 \text { v } 0.8 \pm 0.40 \text { (NS); } \\
\text { Vegetable serves, } 0.1 \pm 0.2 v 0.2 \pm 0.3 \text { (NS) }\end{array}$ & No & - \\
\hline $\begin{array}{l}\text { Zask et al. } \\
2012 \text { [33] }\end{array}$ & $\begin{array}{l}\mathrm{HE} \\
\mathrm{SRM} \\
\mathrm{HEP}\end{array}$ & WNM & NT & $\mathrm{NIC}$ & $\begin{array}{l}\text { Parental food } \\
\text { provision } \\
\text { Direct } \\
\text { lunchbox } \\
\text { observation }\end{array}$ & $\begin{array}{l}\text { Group effect: Intervention } v \text { control at } \\
\text { follow up } \\
\text { Fruit and vegetables serves, } 2.31 \pm 0.11 \mathrm{v} \\
1.73 \pm 0.12(p<0.001) ; \% \text { no EDNP items, } \\
59.0 \pm 4.6 \% \text { v } 44.0 \pm 5.7(p<0.0001)\end{array}$ & Yes & - \\
\hline
\end{tabular}




\begin{tabular}{|c|c|c|c|c|c|c|c|c|}
\hline \multirow[t]{2}{*}{ Reference } & \multirow[t]{2}{*}{$\begin{array}{l}\text { Nutrition } \\
\text { Content }\end{array}$} & \multicolumn{3}{|c|}{ 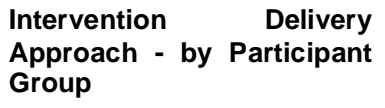 } & \multirow{2}{*}{$\begin{array}{l}\text { Outcome } \\
\text { Outcome } \\
\text { Assessment } \\
\text { Method }\end{array}$} & \multirow[t]{2}{*}{ Main Findings } & \multicolumn{2}{|c|}{$\begin{array}{l}\text { Intervention } \\
\text { Effectiveness }^{a}\end{array}$} \\
\hline & & Staff & Parent & Children & & & End-I & FU \\
\hline $\begin{array}{l}\text { Korenman } \\
\text { et al. } 2013 \\
\text { [47] }\end{array}$ & FR & - & - & - & $\begin{array}{l}\text { Child dietary } \\
\text { intake } \\
\text { Qualitative } \\
\text { mother- } \\
\text { reported FFQ }\end{array}$ & $\begin{array}{l}\text { Group effect: Non-CACFP } \vee \text { CACFP } \\
\text { participants (mean intake) } \\
\text { Milk, }>2 \text { cup/day: } 0.70 \vee 0.73(p<0.05) \\
\text { Fruits or juice, }>3 \text { servings/day: } 0.48 \vee 0.54 \\
(p<0.05) \\
\text { Vegetables, } 2^{+} \text {servings/day: } 0.32 \vee 0.40 \\
\text { (NS) } \\
\text { Salty snacks, } \leq 1-3 \text { servings/wk: } 0.61 \vee 0.59 \\
\text { (NS) } \\
\text { Fast food, } \leq 1-3 \text { servings/wk: } 0.91 \vee 0.87 \\
\text { (p<0.05) } \\
\text { Sweet snacks, } \leq 1-3 \text { servings/wk: } 0.34 \vee \\
0.42 \text { ( }<<0.05) \\
\text { Soda, } \leq 1-3 \text { servings/wk: } 0.74 \vee 0.57 \\
(p<0.05)\end{array}$ & Yes & - \\
\hline $\begin{array}{l}\text { Horne et al. } \\
2011 \text { [39] }\end{array}$ & FMR & - & - & $\mathrm{RMT}+\mathrm{V}$ & $\begin{array}{l}\text { Child dietary } \\
\text { intake } \\
\text { Plate wastage } \\
\text { method (visual } \\
\text { estimation of } \\
\text { leftover food) }\end{array}$ & $\begin{array}{l}\text { Time effect: Snacktime F\&V intake at } \\
\text { baseline and } 6 \text { month follow Up } \\
\text { Target fruit: } 25.3 \%-85.1 \% ; p<0.001 ; d= \\
2.83 \text { ) } \\
\text { Target vegetables: } 24.6-85.1 \% ; p<0.001 ; \\
d=2.59 \text { ), } \\
\text { NB: Similar effects observed for lunchtime } \\
\text { F\&V intake and non-target F\&V }\end{array}$ & Yes & - \\
\hline \multirow[t]{3}{*}{$\begin{array}{l}\text { Bravo et al. } \\
2008 \text { [34] }\end{array}$} & \multirow[t]{3}{*}{$\begin{array}{l}\mathrm{HE} \\
\mathrm{HEP}+\end{array}+$} & \multirow[t]{3}{*}{$\begin{array}{l}\text { NT } \\
\text { WNM }\end{array}$} & \multirow[t]{3}{*}{ WNM } & \multirow[t]{3}{*}{-} & $\begin{array}{l}\text { Staff and } \\
\text { carer } \\
\text { knowledge } \\
\text { Validated 22- } \\
\text { item nutrition } \\
\text { knowledge } \\
\text { questionnaire }\end{array}$ & $\begin{array}{l}\text { Time effect; Baseline v 2-5y } \\
\text { Staff knowledge: All items } p>0.05 \text { ( } n=10 / 24 \\
>75 \% \text { correct at baseline) } \\
\text { Carer knowledge: } 13 \text { areas showed } \\
\text { significant knowledge improvement } \\
(p<0.05), 7 \text { of which related to food safety. } \\
\text { NB: Carers provide care in their own home } \\
\text { for other people's children. Staff coordinate } \\
\text { the scheme. }\end{array}$ & Yes & - \\
\hline & & & & & $\begin{array}{l}\text { Centre food } \\
\text { policies } \\
\text { Study-specific } \\
\text { policy quality } \\
\text { checklist }\end{array}$ & $\begin{array}{l}\text { Time effect: Baseline v 2-5y } \\
\text { Nutrition and Food Policy }(n=7) \text {; Mean } \\
\text { percentage score for all policy categories } \\
\text { post intervention. } 33 \% \text { of centres reached } \\
\text { maximum score }\end{array}$ & Yes & - \\
\hline & & & & & $\begin{array}{l}\text { Centre food } \\
\text { provision } \\
\text { Food provision } \\
\text { history } \\
\text { interview }\end{array}$ & 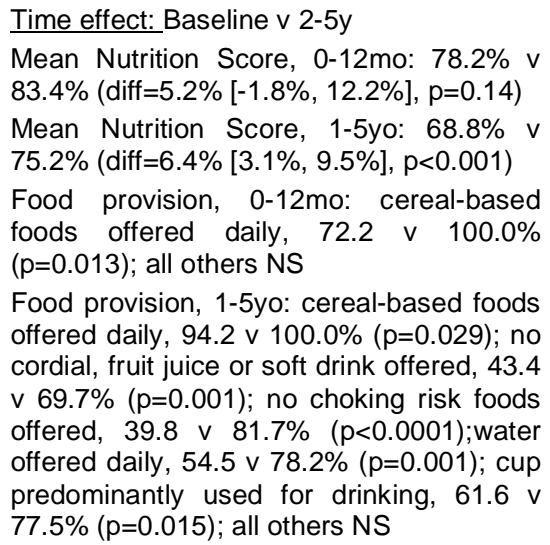 & Yes & - \\
\hline
\end{tabular}

Abbreviations: BM: Behaviour modification (i.e. explicit reporting of inclusion of behaviour modification techniques or strategies such as self-monitoring, goal setting etc.); Ca: Calcium; CR: child reported; DSS: different shaped snacks; End-I: end intervention; EPAO: Environment and Policy Assessment and Observation; FFQ: food frequency questionnaire; FMR: food modelling reward; FSM: food service modification (i.e. updating kitchen, menu assessment); FR: Food Reimbursement; FU: follow up; HE: Healthy eating; HEP: healthy eating/nutrition policy; IF: infant feeding; NIC: nutrition in the curriculum i.e. nutrition-related curriculum activities for children); NS: nutrition skills; MF: modified food provision; NT: nutrition training; PC: Phone Call from trained nutritionist/project staff to provide support; PR: parentreported; RMT: Role Modelling Training; SRM: staff role modelling; SV: site visit (from trained nutritionist,/project staff to observe centre environment); TSE: Teaching Skills Education (i.e. skills to work with parents and/or children regarding nutrition); V: Video (i.e. nutrition education delivered through a video); WG: wholegrain; WNM: written nutrition materials (i.e. pamphlets, written resources; WS: Website (i.e. nutrition education delivered through a website); yr: year.

indicates an intervention effect in at least one outcome measured. 


\section{Primary Outcome: Children's Dietary Intake}

Eight of 11 studies reporting the intervention impact on children's dietary intake showed an effect. Three interventions involving a nutrition curriculum for children, two with a parent component [24, 26] and one without [23], showed significant increases in fruit and vegetables intakes $[23,24,26]$ and meat, dairy and bread consumption [23]. A multi-target intervention did not significantly influence children's fruit or vegetable consumption [25]. An intervention comparing the provision of normal (round, square) versus shaped (heart, hands, animals) high-fibre snacks revealed no significant difference in consumption [41]. Two modelling reward interventions showed promising findings. Use of a video with animated characters led to increased consumption of fruit and vegetables [39]. Training children to role model and encourage fruit and vegetable intake increased short term acceptance under the influence of a girl, but not boy, peer model [40]. A meal and snack reimbursement scheme demonstrated improvements in milk, fruit and fruit juice intake and reductions in consumption of fast food, sweet snacks, and soda [47]. However, no changes in vegetable or salty snack intake were observed [47]. A multi-target Randomised Controlled Trial (RCT) did not influence Latino children's intake of total fat, saturated fat or dietary fibre [28]. The same intervention in predominately 'black' minority groups did not impact on children's total fat or fibre intakes at any time point, but saturated fat intake was significantly lower at one year follow up [27]. Decreases in saturated fat consumption were also observed following a preschool food service modification intervention [44].

\section{Secondary Outcomes}

\section{Centre Environment}

Six studies [34, 35, 37, 43, 45, 46] reported significant changes to the centres nutrition environment. Staff attended workshops that involved a self-assessment of centre nutrition practices pre- and post- nutrition education in three studies [37, 45, 46]. Significant improvements were seen in the centre's nutrition best practices [46] and self-rated nutrition scores $[37,45]$ post-intervention. A study that trained staff resulted in increases in positive discussion around food with children and improvements in food safety practices [35]. Centre's food policies were evaluated in two studies following healthy eating policy education with large improvements post-intervention [34, 43].

\section{Centre Food Provision}

Evaluation of centre-level food provision was undertaken in three studies that trained staff [34, 35, 42]. Significant improvements were observed in the nutritional quality of centres' menus, including the provision of high calcium and moderate iron-containing foods [35], vegetarian meals [35], fresh fruit and vegetables [42], cereal-based foods [34] and water [34], and decreases in the provision of high fat and/or high sugar foods [35], sweetened beverages [34] and choking-risk foods [34].

\section{Parental Food Provision}

Four studies evaluated parental food provision [3133 , 48]. The multi-target 'Lunch is in the bag' intervention involved provision of written education materials to parents and reported significant increases in serves of vegetables and whole grains they provided in children's lunchboxes but no significant difference for fruit at both 6-week [31] and 11-week [32] follow up's. The multi-target 'Tooty Fruity Vegie' intervention that included nutrition workshops for parents resulted in increases in fruit and vegetables, and decreases in unhealthy food and drinks, provided by parents [33]. Additionally one study that didn't include a parent component assessed parental food provision, reporting no change in fruit, vegetables, snacks, or extras provision but decreases in provision of sweetened drinks [48].

\section{Child Knowledge/Attitudes/Preferences}

Two studies examined the provision of training and nutrition materials to staff and a nutrition curriculum to children on children's knowledge [23, 29] and food preferences [23]. Improvements in food and nutrition knowledge were observed overall, with greater improvements for intervention participants compared to controls [29]. Improvements in food identification and recognition, healthy snack identification, willingness to taste foods, and liking of fruit and vegetables were also found [23].

\section{Staff Knowledge/Attitudes/Behaviours}

Nutrition knowledge, attitudes and/or beliefs of early childhood staff were assessed in five studies; four that educated via staff nutrition training [30, 34, 38, 42] and one via a nutrition website [36]. Staff [30, 36, 38] and carer knowledge [34] significantly improved postintervention as did staff attitudes [36, 42] and behaviours $[30,36]$. Importantly, staff in the intervention group felt more at ease post-intervention in 
engaging parents in discussions about their child's eating [42].

\section{DISCUSSION}

This review appraised intervention studies in early childhood settings (ECS) that aim to improve children's nutrition. Twenty-six studies met the review inclusion criteria. Many did not include a control or comparison group and were rated as methodologically weak using a validated quality assessment tool. Most studies demonstrated intervention effectiveness in at least one outcome measured. Eleven studies evaluated the effectiveness of the nutrition intervention by measuring children's dietary intake. Small increases in fruit and vegetable consumption were observed in five studies and decreases in 'discretionary' food or saturated fat intake were seen in three studies. Improvements were also observed in environmental factors that influence children's intake. These findings support the proposition that ECS have good potential as avenues for effective nutrition promotion. However, to date, the strongest evidence relates to the nutrition environment of children in care. Less robust evidence exists on the potential to influence children's dietary intake.

Findings from eleven studies that measured the primary outcome of children's dietary intake were mixed. Six studies (four weak, one moderate) showed a significant intervention effect on children's food or food group intake. Increases in children's short to moderate term fruit or vegetable intake (i.e. up to 6 months), of 0.1 [25] to 1.9 [23] serves, were observed. These results are consistent with those observed from nutrition education interventions [49]. One study, although rated weak, demonstrated increases in fruit and vegetable snack consumption which was sustained three months post-intervention [26]. Discretionary food consumption was measured in one study, with decreases observed [47]. Two studies demonstrated a significant intervention effect on children's nutrient intake. A two-year RCT was not effective in reducing Latino pre-schoolers' fat or fibre intakes, but did result in decreases in saturated fat intake in 'black' preschoolers [27], whilst decreases in saturated fat consumption also resulted from a food service modification intervention [44]. Despite these studies rating poorly, the findings are promising and highlight that ECS nutrition promotion interventions can positively influence children's nutrition intake.

Nine studies evaluated individual or environmental influences on dietary intake as measures of intervention impact or proxy for children's consumption, such as centre or parental food provision, child, parent or staff knowledge, attitudes or behaviours, and measures of the ECS nutrition environment or policy. Of the seven studies that reported on parental or ECS food provision, nearly all $(n=6)$ reported positive changes across a number of food groups. For example, small improvements in the vegetable $(0.1$ to 0.3 serves), but not fruit content of children's lunch boxes were observed in three controlled clinical trials (CCT). Improvements in whole grain and sweetened beverage provision by parents were also reported. Centres' menus improved across a range of food groups in two cohort studies and one CCT. Scores on a policy or environment checklist (either directly observed or selfreported) showed improvements ranging from $10-50 \%$. Significant improvements in child, parent or staff knowledge, attitudes or behaviours were observed consistently across studies that included these measures. Improvements were evident only in the intervention group in the three studies that included a comparison group. Collectively, these results suggest that environmental interventions in ECS can achieve improvements in determinants of children's dietary intake.

Overall, this review highlights that limited evidence is available on the ability of ECS nutrition promotion interventions to influence children's dietary intake, in contrast to the stronger literature on their potential to influence the ECS nutrition environment. Larson and colleagues (2010) [18] recently reviewed the evidence on child care state regulations, policies and practices, and interventions for promoting healthy eating and physical activity in preschool children attending child care. Positive intervention effects were found for evaluations of child-care practices or policies, children's mealtime behaviours, dietary preferences or dietary quality, and obesity risk [18]. This review differed from the present review as included studies were those with implications for preventing obesity and studies beyond the United States context were not included [18]. Nonetheless, our findings align with those by Larson and colleagues [18] and support social-ecological theories of behaviour change which highlight the importance of both individual and environmental determinants of children's dietary behaviours [50]. Thus, our finding that ECS interventions can modify environmental and behavioural determinants of children's food intake signals that intervention development should continue as a priority to inform policy and practice. 
Whilst there is considerable progress being made in increasing our understanding of the potential of ECS in improving children's dietary intake, this review demonstrates that much remains to be learned. Despite the intended outcome of ECS nutrition interventions being an improvement in children's food and/or nutrient intake, less than half $(n=11 / 26)$ of studies in this review included measures of this primary outcome of interest. In addition, although most interventions promoted food intake in line with dietary guidelines, evaluation of the impact on food or food group intake beyond fruit and vegetables was limited $(n=2)$. Similarly, evaluation of the impact on nutrient intake beyond fat and fibre was not conducted. Given children's high intake of milk [15, 51], which can displace other food group intake, reducing variety and increasing risk of iron deficiency [52], as well as children's high consumption of discretionary foods, saturated fat, added sugars and sodium in Western countries $[15,16,53]$, the intervention impact on these foods and nutrients needs further evaluation. The limited inclusion of dietary outcomes in studies ( 40\%) to date is likely to reflect both the difficulty in accurately measuring children's food intake and the newness of ECS as a setting for nutrition promotion, where the objective of studies may be to assess the ECS capacity for environmental, policy or menu change prior to assessment of dietary intake. This highlights that assessment beyond evaluation of the nutrition environment, to inclusion of children's nutritional intake, is required.

Future research requires the design of interventions with strong methodological quality. One third $(n=9)$ of studies included in this review did not include a control or comparison group ( $n=6$ rated weak) and of the 18 studies that did, half $(n=9)$ were rated as methodologically weak using a validated quality assessment tool [21]. Where possible, the use of controlled trial or quasi-experimental designs needs to be prioritised to strengthen the evidence-base of this area. At a minimum, more attention to intervention fidelity, addressing selection and measurement bias, and management of potential confounding in data analysis is needed to provide greater confidence in study findings [21]. Further, while environmental and behavioural measures are important, until intervention outcomes in terms of children's dietary intake and health status are consistently and comprehensively evaluated, the utility of ECS interventions as effective public health nutrition approaches will remain unclear.
A final area for consideration is the area of refinement and innovation in intervention design. Some of the promising studies reviewed aimed to evaluate the effectiveness of innovative nutrition or behavioural strategies in isolation [39-41]. This type of research is needed to improve the effectiveness of comprehensive interventions and programs, which to date have inconsistent or small effects on measured outcomes. By selecting the behavioural targets, intervention components and strategies based on theoretical or empirical evidence, there is potential to improve intervention effectiveness [54]. Careful attention to the development and testing of intervention content could also lead to effective but less intense interventions better suited to delivery in public health settings.

A review strength is the inclusion of a broad range of study designs which allowed a critique of the range of ECS interventions which form the current literature base. However inclusion of studies which vary in methodological quality, intervention and follow up duration does make comparison between studies difficult and the conclusions that can be drawn need to be with caution. However the use of a validated Quality Assessment Tool [21] is a review strength and makes the quality of included studies transparent. Nonetheless, as evident in this review, this assessment tool can result in a similar quality grading for both prepost studies and RCT's. The absence of a second reviewer in screening review articles and the lack of consideration of the grey literature are also limitations as they increase potential bias. Lastly, as the majority of studies reviewed were conducted in the United States, adaptations would be required to ensure they are culturally appropriate and generalizable to the other countries.

\section{CONCLUSION}

In conclusion, this review identified that early childhood setting interventions can achieve changes in children's dietary intake and associated socioenvironmental determinants. Yet few ECS intervention studies have evaluated the impact on children's intake beyond fruit and vegetable, or saturated fat, consumption. With the body of evidence in this research field building, researchers should continue to move beyond nutrition environment measures only and prioritise inclusion of dietary outcomes, including a range of measures, as part of their evaluation of ECS nutrition interventions. Future research should also attend to the quality of studies so that we can be sure that intervention effects are not due to bias, chance or 
confounding and findings are generalizable. Future intervention development needs to carefully consider the behavioural targets, modifiable determinants and utilise age-appropriate and effective behaviour change theory. This review provides a benchmark for future intervention development and study design to answer the question of how children's nutrition can be positively influenced through early childhood settings.

\section{ACKNOWLEDGEMENTS}

RKG was supported by a National Heart Foundation Fellowship (478115). Support for LKB included funds from a Fellowships SA grant.

\section{CONFLICTS OF INTEREST}

The authors declare no conflict of interest.

\section{REFERENCES}

[1] Lynch JW, Davey-Smith G. A life-course aproach to chronic disease epidemiology. Annu Rev Public Health 2005; 26: 135.

http://dx.doi.org/10.1146/annurev.publhealth.26.021304.1445 $\underline{05}$

[2] Wu TC, Chen PH. Health consequences of nutrition in childhood and early infancy. Pediatr Neonatol 2009; 50: 13542.

\section{http://dx.doi.org/10.1016/S1875-9572(09)60051-6}

[3] Morgan J. Nutrition for toddlers: the foundation for good health--1. toddlers' nutritional needs: what are they and are they being met? J Fam Health Care 2005; 15: 56-9.

[4] Pan American Health Organization (PAHO)/World Health Organization (WHO). Guiding principles for complementary feeding of the breastfed child. PAHO/WHO. Washington, DC: 2003.

[5] Birch L, Savage JS, Ventura A. Influences on the Development of Children's Eating Behaviours: From Infancy to Adolescence. Can J Diet Pract Res 2007; 68: s1-s56.

[6] Devine CM. A life course perspective: understanding food choices in time, social location, and history. J Nutr Educ Behav 2005; 37: 121-8.

http://dx.doi.org/10.1016/S1499-4046(06)60266-2

[7] Golley RK, Hendrie A, Slater A, et al. Interventions that involve parents to improve children's weight-related nutrition intake and activity patterns - what nutrition and activity targets and behaviour change techniques are associated with intervention effectiveness? Obes Rev 2011; 12: 114-30. http://dx.doi.org/10.1111/j.1467-789X.2010.00745.x

[8] Kaphingst KM, Story M. Child care as an untapped setting for obesity prevention: state child care licensing regulations related to nutrition, physical activity, and media use for preschool-aged children in the United States. Prev Chronic Dis 2009; 6: A11.

[9] Kalb G, Lee W. Childcare Use and Parents' Labour Supply in Australia. Research Report, 2007. Melbourne Institute of Applied Economic and Social Research. Victoria, Australia. http://hdl.handle.net/10620/3329.

[10] Pollard C, Lewis J. Food service in long day care centres - an opportunity for public health intervention. Aust N Z J Public Health 1999; 23: 606-10.

http://dx.doi.org/10.1111/j.1467-842X.1999.tb01545.x
[11] Sigman-Grant M, Christiansen E, Branen L, et al. About feeding children: mealtimes in child-care centers in four western states. J Am Diet Assoc 2008; 108: 340-6. http://dx.doi.org/10.1016/i.jada.2007.09.006

[12] Ball SC, Benjamin SE, Ward DS. Dietary intakes in North Carolina child-care centers: Are children meeting current recommendations? J Am Diet Assoc 2008; 108: 718-21. http://dx.doi.org/10.1016/j.jada.2008.01.014

[13] Erinosho T, Dixon LB, Young C, et al. Nutrition practices and children's dietary intakes at 40 child-care centers in New York City. J Am Diet Assoc 2011; 111: 1391-7. http://dx.doi.org/10.1016/j.jada.2011.06.001

[14] Briley ME, Jastrow S, Vickers J, et al. Dietary intake at childcare centers and away: Are parents and care providers working as partners or at cross-purposes? J Am Diet Assoc 1999; 99: 950-4.

http://dx.doi.org/10.1016/S0002-8223(99)00226-6

[15] Commonwealth Scientific and Industrial Research Organisation. 2007 Australian National Children's Nutrition and Physical Activity Survey: Main Findings. Commonwealth of Australia. Canberra: 2008.

[16] Siega-Riz AM, Kinlaw A, Deming DM, et al. New findings from the Feeding Infants and Toddlers Study 2008. Nestle Nutr Workshop Ser Pediatr Program 2011; 68: 83-100 discussion -5 .

[17] Craigie AM, Lake AA, Kelly SA, et al. Tracking of obesityrelated behaviours from childhood to adulthood: A systematic review. Maturitas 2011; 70: 266-84.

http://dx.doi.org/10.1016/j.maturitas.2011.08.005

[18] Larson N, Ward DS, Neelon SB, et al. What role can childcare settings play in obesity prevention? A review of the evidence and call for research efforts. J Am Diet Assoc 2011; 111: 1343-62.

http://dx.doi.org/10.1016/j.jada.2011.06.007

[19] Story M, Kaphingst K, French S. The role of child care settings in obesity prevention. Future Child 2006; 16: 143-68. http://dx.doi.org/10.1353/foc.2006.0010

[20] Erinosho TO, Hales DP, McWilliams CP, et al. Nutrition policies at child-care centers and impact on role modeling of healthy eating behaviors of caregivers. J Acad Nutr Diet 2012; 112: 119-24

http://dx.doi.org/10.1016/j.jada.2011.08.048

[21] Thomas BH, Ciliska D, Dobbins $M$, et al. A process for systematically reviewing the literature: providing the research evidence for public health nursing interventions. Worldviews Evid Based Nurs 2004; 1: 176-84. http://dx.doi.org/10.1111/i.1524-475X.2004.04006.x

[22] Deeks JJ, Dinnes J, D'Amico R, et al. Evaluating nonrandomised intervention studies. Health Technol Assess 2003; 7: 1-186.

http://dx.doi.org/10.3310/hta7270

[23] Cason KL. Evaluation of a preschool nutrition education program based on the theory of multiple intelligences. J Nutr Educ 2001; 33: 161-4. http://dx.doi.org/10.1016/S1499-4046(06)60186-3

[24] De Bock F, Breitenstein L, Fischer JE. Positive impact of a pre-school-based nutritional intervention on children's fruit and vegetable intake: results of a cluster-randomized trial. Public Health Nutr 2012; 15: 466-75. http://dx.doi.org/10.1017/S136898001100200X

[25] Sharma S, Ru-Jye C, Hedberg AM. Pilot-testing catch early childhood: a preschool-based healthy nutrition and physical activity program. Am J Health Educ 2011; 42: 12-23. http://dx.doi.org/10.1080/19325037.2011.10599169

[26] Witt KE, Dunn C. Increasing fruit and vegetable consumption among preschoolers: evaluation of color me healthy. J Nutr Educ Behav 2012; 44: 107-13.

http://dx.doi.org/10.1016/i.jneb.2011.01.002 
[27] Fitzgibbon ML, Stolley MR, Schiffer L, et al. Two-year followup results for Hip-Hop to Health Jr.: a randomized controlled trial for overweight prevention in preschool minority children. J Pediatr 2005; 146: 618-25.

http://dx.doi.org/10.1016/j.jpeds.2004.12.019

[28] Fitzgibbon ML, Stolley MR, Schiffer L, et al. Hip-Hop to Health Jr. for Latino preschool children. Obesity (Silver Spring) 2006; 14: 1616-25. http://dx.doi.org/10.1038/oby.2006.186

[29] Gorelick MC, Clark AE. Effects of a nutrition program on knowledge of preschool children. J Nutr Educ Behav 1985; 17: 88-92.

http://dx.doi.org/10.1016/S0022-3182(85)80210-7

[30] Herman A, Nelson BB, Teutsch C, et al. "Eat Healthy, Stay Active!": a coordinated intervention to improve nutrition and physical activity among Head Start parents, staff, and children. Am J Health Promot 2012; 27: e27-36. http://dx.doi.org/10.4278/ajhp.110412-QUAN-157

[31] Briley ME, Ranjit N, Holescher DM, et al. Unbundling Outcomes of a Multilevel Intervention to Increase Fruit, Vegetables and Whole Grains Parents Pack for their Preschool Children in Sack Lunches. Am J Health Educ 2012; 43: 135-42.

\section{http://dx.doi.org/10.1080/19325037.2012.10599230}

[32] Sweitzer SJ, Briley ME, Roberts-Gray C, et al. Lunch is in the bag: increasing fruits, vegetables, and whole grains in sack lunches of preschool-aged children. J Am Diet Assoc 2010; 110: 1058-64.

http://dx.doi.org/10.1016/j.jada.2010.04.010

[33] Zask A, Adams JK, Brooks LO, et al. Tooty Fruity Vegie: an obesity prevention intervention evaluation in Australian preschools. Health Promot J Austr 2012; 23: 10-5.

[34] Bravo A, Cass Y, Tranter D. Good food in family day care: Improving nutrition and food safety in family day care. Aus $\mathrm{J}$ Nutr Diet 2008; 65: 47-55.

http://dx.doi.org/10.1111/j.1747-0080.2007.00136.x

[35] Sangster J, Eccelston P, Stickney B. Improving what's in the lunchbox in childcare centres. Health Promot J Austr 2003; 14: 171.

[36] Clark A, Anderson J, Adams E, et al. Assessing an infant feeding web site as a nutrition education tool for child care providers. J Nutr Educ Behav 2009; 41: 41-6. http://dx.doi.org/10.1016/j.jneb.2007.12.007

[37] Ward DS, Benjamin SE, Ammerman AS, et al. Nutrition and physical activity in child care: results from an environmental intervention. Am J Prev Med 2008; 35: 352-6. http://dx.doi.org/10.1016/j.amepre.2008.06.030

[38] Benjamin SE, Tate DF, Bangdiwala SI, et al. Preparing Child Care Health Consultants to address childhood overweight: a randomized controlled trial comparing web to in-person training. Matern Child Health J 2008; 12: 662-9. http://dx.doi.org/10.1007/s10995-007-0277-1

[39] Horne PJ, Greenhalgh J, Erjavec M, et al. Increasing preschool children's consumption of fruit and vegetables. A modelling and rewards intervention. Appetite 2011; 56: 37585.

http://dx.doi.org/10.1016/j.appet.2010.11.146

[40] Hendy HM. Effectiveness of trained peer models to encourage food acceptance in preschool children. Appetite 2002; 39: 217-25.

http://dx.doi.org/10.1006/appe.2002.0510

[41] Boyer LE, Laurentz S, McCabe GP, et al. Shape of snack foods does not predict snack intake in a sample of preschoolers: a cross-over study. Int J Behav Nutr Phys Act 2012; 9: 94.

http://dx.doi.org/10.1186/1479-5868-9-94

[42] Gosliner WA, James P, Yancey AK, et al. Impact of a worksite wellness program on the nutrition and physical activity environment of child care centers. Am $\mathrm{J}$ Health Promot 2010; 24: 186-9. http://dx.doi.org/10.4278/ajhp.08022719

[43] Matwiejczyk L, McWhinnie JA, Colmer K. An evaluation of a nutrition intervention at childcare centres in South Australia. Health Promot J Austr 2007; 18: 159-62.

[44] Williams CL, Bollella MC, Strobino BA, et al. "Healthy-start": outcome of an intervention to promote a heart healthy diet in preschool children. J Am Coll Nutr 2002; 21: 62-71. http://dx.doi.org/10.1080/07315724.2002.10719195

[45] Benjamin SE, Ammerman A, Sommers J, et al. Nutrition and physical activity self-assessment for child care (NAP SACC): results from a pilot intervention. J Nutr Educ Behav 2007; 39: 142-9.

http://dx.doi.org/10.1016/j.jneb.2006.08.027

[46] Drummond RL, Staten LK, Sanford MR, et al. A pebble in the pond: the ripple effect of an obesity prevention intervention targeting the child care environment. Health Promot Pract 2009; 10: 156S-67S http://dx.doi.org/10.1177/1524839908331267

[47] Korenman S, Abner KS, Kaestner R, et al. The Child and Adult Care Food Program and the Nutrition of Preschoolers. Early Child Res Q 2013; 28: 325-36. http://dx.doi.org/10.1016/j.ecresq.2012.07.007

[48] Hardy LL, King L, Kelly B, et al. Munch and Move: evaluation of a preschool healthy eating and movement skill program. Int J Behav Nutr Phys Act 2010; 7: 80 http://dx.doi.org/10.1186/1479-5868-7-80

[49] Buttriss J, Stanner S, McKevith B, et al. Successful ways to modify food choice: lessons from the literature. Nutr Bull 2004; 29: 333-43.

http://dx.doi.org/10.1111/j.1467-3010.2004.00462.x

[50] McLeroy KR, Bibeau D, Steckler A, et al. An ecological perspective on health promotion programs. Health Educ Behav 1988; 15: 351-77. http://dx.doi.org/10.1177/109019818801500401

[51] Thane CW, Walmsley CM, Bates CJ, et al. Risk factors for poor iron status in British toddlers: further analysis of data from the National Diet and Nutrition Survey of children aged 1.5-4.5 years. Public Health Nutr 2000; 3: 433-40. http://dx.doi.org/10.1017/S1368980000000501

[52] Ziegler EE. Consumption of cow's milk as a cause of iron deficiency in infants and toddlers. Nutr Rev 2011; 69 Suppl 1 : S37-42. http://dx.doi.org/10.1111/j.1753-4887.2011.00431.x

[53] Bates B, Lennox A, Swan G, editors. National Diet and Nutrition Survey: Headline results from Year 1 of the Rolling Programme (2008/2009). United Kingdom: Department of Health and the Food Standards Agency; 2010 [cited October 2014]; Available from: http://multimedia.food.gov.uk/ multimedia/pdfs/publication/ndnsreport0809.pdf

[54] Brug J, Oenema A, Ferreira I. Theory, evidence and Intervention Mapping to improve behavior nutrition and physical activity interventions. Int $\mathrm{J}$ Behav Nutr Phys Act 2005; 2: 2. http://dx.doi.org/10.1186/1479-5868-2-2 